\title{
THE COMPLEXITY OF LEARNING SUBSEQ $(A)$
}

\author{
STEPHEN FENNER, WILLIAM GASARCH, AND BRIAN POSTOW
}

\begin{abstract}
Higman essentially showed that if $A$ is any language then $\operatorname{SUBSEQ}(A)$ is regular, where $\operatorname{SUBSEQ}(A)$ is the language of all subsequences of strings in $A$. Let $s_{1}, s_{2}, s_{3}, \ldots$ be the standard lexicographic enumeration of all strings over some finite alphabet. We consider the following inductive inference problem: given $A\left(s_{1}\right), A\left(s_{2}\right)$, $A\left(s_{3}\right), \ldots$, learn, in the limit, a DFA for $\operatorname{SUBSEQ}(A)$. We consider this model of learning and the variants of it that are usually studied in Inductive Inference: anomalies, mindchanges, teams, and combinations thereof

This paper is a significant revision and expansion of an earlier conference version [10].
\end{abstract}

S1. Introduction. Our work is based on a remarkable theorem of Higman $[22],{ }^{1}$ given below as Theorem 1.3.

Convention: $\Sigma$ is a finite alphabet.

Definition 1.1. Let $x, y \in \Sigma^{*}$. We say that $x$ is a subsequence of $y$ if $x=$ $x_{1} \cdots x_{n}$ and $y \in \Sigma^{*} x_{1} \Sigma^{*} x_{2} \cdots x_{n-1} \Sigma^{*} x_{n} \Sigma^{*}$. We denote this by $x \preceq y$.

Notation 1.2. If $A$ is a set of strings, then $\operatorname{SUBSEQ}(A)$ is the set of subsequences of strings in $A$.

Higman [22] showed the following using well-quasi-order theory.

Theorem 1.3 (Higman [22]). If $A$ is any language over $\Sigma^{*}$, then $\operatorname{SUBSEQ}(A)$ is regular. In fact, for any language $A$ there is a unique minimum (and finite) set $S$ of strings such that

$$
\operatorname{SUBSEQ}(A)=\left\{x \in \Sigma^{*}:(\forall z \in S)[z \npreceq x]\right\} .
$$

The original proof of this theorem is nonconstructive. Nerode's Recursive Mathematics Program [27, 9] attempts to pin down what it means for a proof to be noneffective. In [11] we showed that there can be no effective proof. In particular we showed (among other things) that there is no partial computable

Key words and phrases. machine learning, inductive inference, automata, computability, subsequence.

The first author is partially supported by NSF grant CCF-05-15269.

The second author is partially supported by NSF grant CCR-01-05413.

Some of this work was done while the third author was at Union College, Schenectady, NY.

${ }^{1}$ The result we attribute to Higman is actually an easy consequence of his work. See [11] for more discussion. 
function that takes as input an index for a Turing Machine deciding $A$ and outputs a DFA for $\operatorname{SUBSEQ}(A)$.

What if $A$ is not decidable? Then we cannot be given a Turing machine deciding $A$. We could instead be given $A$ one string at a time. In this case we can try to learn a DFA for $\operatorname{SUBSEQ}(A)$ in the limit. We use variations of notions from Inductive Inference to formalize this.

S2. Inductive Inference and our variants. In Inductive Inference [5, 7, $20]$ the basic model of learning is as follows.

Definition 2.1. A class $\mathcal{A}$ of decidable sets of strings ${ }^{2}$ is in EX if there is a Turing machine $M$ (the learner) such that if $M$ is given $A(\varepsilon), A(0), A(1), A(00)$, $A(01), A(10), A(11), A(000), \ldots$, where $A \in \mathcal{A}$, then $M$ will output $e_{1}, e_{2}, e_{3}, \ldots$ such that $\lim _{s} e_{s}=e$ and $e$ is an index for a Turing machine that decides $A$.

Note that the set $A$ must be computable and the learner learns a program deciding it. There are variants $[3,16,18]$ where the set need not be computable and the learner learns something about the set (e.g., "Is it infinite?" or some other question). Our work is in the same spirit in that we will be given the characteristic sequence of a set $A$ and try to learn $\operatorname{SUBSEQ}(A)$.

Notation 2.2. We let $s_{1}, s_{2}, s_{3}, \ldots$ be the standard length-first lexicographic enumeration of $\Sigma^{*}$. We refer to Turing machines as TMs.

Definition 2.3. A class $\mathcal{A}$ of sets of strings in $\Sigma^{*}$ is in SUBSEQ-EX if there is a TM $M$ (the learner) such that if $M$ is given $A\left(s_{1}\right), A\left(s_{2}\right), A\left(s_{3}\right), \ldots$ where $A \in \mathcal{A}$, then $M$ will output $e_{1}, e_{2}, e_{3}, \ldots$ such that $\lim _{s} e_{s}=e$ and $e$ is an index for a DFA that recognizes $\operatorname{SUBSEQ}(A)$. It is easy to see that we can take $e$ to be the least index of the minimum-state DFA that recognizes $\operatorname{SUBSEQ}(A)$. Formally, we will refer to $A\left(s_{1}\right) A\left(s_{2}\right) A\left(s_{3}\right) \cdots$ as being on an auxiliary tape.

Remark. Definitions 2.1 and 2.3 are similar in that the full characteristic function of a language is provided to the learner. In an alternate style of inductive inference, the learner is provided instead with a list of elements ("positive examples") of a c.e. ${ }^{3}$ language in some arbitrary order, and the learner must converge to a grammar (equivalently, a c.e. index) for the language [20]. Although it is not the focus of our current investigation, we do give some basic observations in Section 5.2 about learning $\operatorname{SUBSEQ}(A)$ from one-sided data, both positive and negative.

Remark. In Definition 2.3 the learner gets $A$ on its tape, not $\operatorname{SUBSEQ}(A)$, but still must learn a DFA for $\operatorname{SUBSEQ}(A)$. One might ask whether it makes more sense to learn $\operatorname{SUBSEQ}(A)$ with $\operatorname{SUBSEQ}(A)$ on the tape instead of $A$. The answer is no, at least for standard SUBSEQ-EX-learning; Proposition 4.18 gives a single learner that would learn $\operatorname{SUBSEQ}(A)$ this way for all $A \subseteq \Sigma^{*}$. Even with more restricted learning variants (e.g., bounded mind-changes, one-sided

\footnotetext{
${ }^{2}$ The basic model is usually described in terms of learning computable functions; however, virtually all of the results hold in the setting of decidable sets.

${ }^{3}$ C.e. stands for "computably enumerable," which is synonymous with "recursively enumerable."
} 
data), learning from $\operatorname{SUBSEQ}(A)$ offers nothing new, as it is merely equivalent to learning from $A$ when we restrict ourselves to languages $A$ such that $A=$ $\operatorname{SUBSEQ}(A)$ (the " $\preceq$-closed" languages of Definition 4.10, below).

We give examples of elements of SUBSEQ-EX in Section 4.3, where we show that SUBSEQ-EX contains the class of all finite languages. More generally, SUBSEQ-EX contains the class of all regular languages and-more generally still - the class of all context-free languages. Additional examples are given in Section 5.1 (Proposition 5.2) and Section 6.

This problem is part of a general theme of research: given a language $A$, rather than try to learn a program to decide it (which is not possible if $A$ is undecidable) learn some aspect of it. In this case we learn $\operatorname{SUBSEQ}(A)$. Note that we learn $\operatorname{SUBSEQ}(A)$ in a very strong way in that we have a DFA for it. One can certainly consider learning other devices for SUBSEQ $(A)$ (e.g., context-free grammars, polynomial-time machines, etc.) With respect to the basic model of inductive inference, it turns out that the type of device being learned is largely irrelevant: learning a DFA for $\operatorname{SUBSEQ}(A)$ is equivalent to learning a total Turing machine for SUBSEQ $(A)$. We will say more about this in Section 5.2.

If $\mathcal{A} \in \mathrm{EX}$, then a TM can infer a program that decides any $A \in \mathcal{A}$. That program is useful if you want to determine membership of particular strings, but not useful if you want most global properties (e.g., "Is $A$ infinite?"). If $\mathcal{A} \in$ SUBSEQ-EX, then a TM can infer a DFA for $\operatorname{SUBSEQ}(A)$. The DFA is useful if you want to determine virtually any property of $\operatorname{SUBSEQ}(A)$ (e.g., "Is SUBSEQ $(A)$ infinite?") but not useful if you want to answer almost any question about $A$.

S3. Summary of main results. We look at anomalies, mind-changes, and teams, both alone and in combination. These are standard variants of the usual model in inductive inference. See [7] and [32] for the definitions within inductive inference; however, our definitions are similar.

We list definitions and our main results. All are related to the EX-style of learning (Definitions 2.1 and 2.3) -learning from complete data about $A$, i.e., its characteristic function. Section 5.2 discusses other styles of learning.

1. Let $\mathcal{A} \in$ SUBSEQ-EX ${ }^{a}$ mean that the final DFA may be wrong on at most $a$ strings (called anomalies). Also let $\mathcal{A} \in \mathrm{SUBSEQ}-\mathrm{EX}^{*}$ mean that the final DFA may be wrong on a finite number of strings (i.e., a finite number of anomalies - the number perhaps varying with $A$ ). The anomaly hierarchy collapses; that is,

$$
\text { SUBSEQ-EX }=\text { SUBSEQ-EX* }
$$

This contrasts sharply with the case of $\mathrm{EX}^{a}$, where it was proven in [7] that $\mathrm{EX}^{a} \subset \mathrm{EX}^{a+1}$.

2. Let $\mathcal{A} \in \mathrm{SUBSEQ}-\mathrm{EX}_{n}$ mean that the TM makes at most $n+1$ conjectures (and hence changes its mind at most $n$ times). The mind-change hierarchy separates; that is, for all $n$,

$$
\text { SUBSEQ-EX }{ }_{n} \subset \text { SUBSEQ-EX }{ }_{n+1} \text {. }
$$

This is analogous to the result proved in [7]. 
3. The mind-change hierarchy also separates if you allow a transfinite number of mind-changes, up to $\omega_{1}^{\mathrm{CK}}$ (see "Transfinite Mind Changes and Procrastination" in Section 5.4). This is also analogous to the result in [13].

4. Let $\mathcal{A} \in[a, b]$ SUBSEQ-EX mean that there is a team of $b$ TMs trying to learn the DFA, and we demand that at least $a$ of them succeed (it may be a different $a$ machines for different $A \in \mathcal{A}$ ).

(a) Analogous to results in $[28,29]$, if $1 \leq a \leq b$ and $q=\lfloor b / a\rfloor$, then

$$
[a, b] \mathrm{SUBSEQ}-\mathrm{EX}=[1, q] \mathrm{SUBSEQ}-\mathrm{EX} .
$$

Hence we need only look at the team learning classes $[1, n]$ SUBSEQ-EX. (b) The team hierarchy separates. That is, for all $b$,

$$
[1, b] \text { SUBSEQ-EX } \subset[1, b+1] \text { SUBSEQ-EX }
$$

These are also analogous to results from [32].

5 . In contrast with results in [32], the anomaly hierarchy collapses in the presence of teams. That is, for all $1 \leq a \leq b$,

$$
[a, b] \mathrm{SUBSEQ}-\mathrm{EX}^{*}=[a, b] \mathrm{SUBSEQ}-\mathrm{EX} .
$$

6. There are no trade-offs between bounded anomalies and mind-changes:

$$
\mathrm{SUBSEQ}_{\mathrm{EX}}^{a}=\mathrm{SUBSEQ}_{c} \mathrm{EX}_{c}
$$

for all $a$ and $c$. This result contrasts with [7, Theorem 2.14]. However, SUBSEQ-EX ${ }_{0}^{*} \nsubseteq \bigcup_{c \in \mathbb{N}}$ SUBSEQ-EX $_{c}$, and for any $c>0$, SUBSEQ-EX ${ }_{c} \nsubseteq$ SUBSEQ-EX $X_{c-1}^{*}$, analogous to $[7$, Theorem 2.16]. There are nontrivial trade-offs between anomaly revisions (transfinite anomalies) and mindchanges.

7. There are several interesting trade-offs between mind-changes and teams. For all $1 \leq a \leq b$ and $c \geq 0$,

$$
[a, b] \mathrm{SUBSEQ}_{\mathrm{EX}} \subseteq[1,\lfloor b / a\rfloor] \mathrm{SUBSEQ}-\mathrm{EX}_{b(c+1)-1} .
$$

This is also the case for EX, as described by Jain [23] - see Appendix A. (Somewhat to the converse, it is easily seen that $[1, q]$ SUBSEQ-EX $_{c} \subseteq$ $[a, a q]$ SUBSEQ-EX ${ }_{c}$ for $q \geq 1$.) Also, analogously to [32, Theorems 4.1, 4.2],

$$
\mathrm{SUBSEQ} \mathrm{EX}_{b(c+1)-1} \subseteq[1, b] \mathrm{SUBSEQ}-\mathrm{EX}_{c} \nsupseteq \mathrm{SUBSEQ} \mathrm{EX}_{b(c+1)} .
$$

In the other direction, however, the analogy is curiously off by a factor of two: if $b>1$ and $c \geq 1$, then

$$
\mathrm{SUBSEQ} \mathrm{EX}_{2 b(c+1)-3} \supseteq[1, b] \mathrm{SUBSEQ}_{\mathrm{EX}} \nsubseteq \mathrm{SUBSEQ}_{c} \mathrm{EX}_{2 b(c+1)-4} \text {. }
$$

Note 3.1. PEX [6, 7] is like EX except that even the intermediate conjectures must be for total TMs. The class SUBSEQ-EX is similar in that all the machines are total (in fact, DFAs) but different in that we learn the subsequence language, and the input need not be computable. The anomaly hierarchy for SUBSEQ-EX collapses just as it does for PEX; however, the team hierarchy for SUBSEQ-EX is proper, unlike for PEX. 


\section{S4. Definitions and first results.}

Notation 4.1. We let $\mathbb{N}=\{0,1,2, \ldots\}$ and $\mathbb{N}^{+}=\{1,2,3, \ldots\}$. We assume that $\Sigma$ is some finite alphabet, that $0,1 \in \Sigma$, and that all languages are subsets of $\Sigma^{*}$. We identify a language with its characteristic function.

Notation 4.2. For $n \in \mathbb{N}$, we let $\Sigma^{=n}$ denote the set of all strings over $\Sigma$ of length $n$. We also define $\Sigma^{\leq n}=\bigcup_{i \leq n} \Sigma^{=i}$ and $\Sigma^{<n}=\bigcup_{i<n} \Sigma^{=i}$. $\Sigma^{\geq n}$ and $\Sigma^{>n}$ are defined analogously.

4.1. Classes of languages. We define classes of languages via the types of machines that recognize them.

Notation 4.3 .

1. $D_{1}, D_{2}, \ldots$ is a standard enumeration of finite languages. ( $e$ is the canonical index of $D_{e}$.)

2. $F_{1}, F_{2}, \ldots$ is a standard enumeration of minimized DFAs, presented in some canonical form so that for all $i \neq j$ we have $L\left(F_{i}\right) \neq L\left(F_{j}\right)$. Let REG $=$ $\left\{L\left(F_{1}\right), L\left(F_{2}\right), \ldots\right\}$.

3. $P_{1}, P_{2}, \ldots$ is a standard enumeration of $\{0,1\}$-valued polynomial-time TMs. Let $\mathrm{P}=\left\{L\left(P_{1}\right), L\left(P_{2}\right), \ldots\right\}$. Note that these machines are total.

4. $M_{1}, M_{2}, \ldots$ is a standard enumeration of Turing Machines. We let $\mathrm{CE}=$ $\left\{L\left(M_{1}\right), L\left(M_{2}\right), \ldots\right\}$, where $L\left(M_{i}\right)$ is the set of all $x$ such that $M_{i}(x)$ halts with output 1 (i.e., $M_{i}(x)$ accepts). We define $W_{i}:=L\left(M_{i}\right)$ and say that $i$ is a c.e. index of $W_{i}$. For $n \in \mathbb{N}$ we let $W_{i, n} \subseteq W_{i}$ be the result of running some standard uniformly computable enumeration of $W_{i}$ for $n$ steps.

5. We let $\mathrm{DEC}=\{L(N): N$ is a total TM $\}$.

The notation below is mostly standard. For the notation that relates to computability theory, our reference is [33].

For separation results, we will often construct tally sets, i.e., subsets of $0^{*}$.

Notation 4.4 .

1. The empty string is denoted by $\varepsilon$.

2. For $m \in \mathbb{N}$, we define $0^{<m}=\left\{0^{i}: i<m\right\}$.

3. If $B \subseteq 0^{*}$ is finite, we let $m(B)$ denote the least $m$ such that $B \subseteq 0^{<m}$, and we observe that $\operatorname{SUBSEQ}(B)=0^{<m(B)}$.

4. If $A$ is a set then $\mathcal{P}(A)$ is the powerset of $A$.

Notation 4.5. If $B, C \subseteq 0^{*}$ and $B$ is finite, we define a "shifted join" of $B$ and $C$ as follows:

$$
B \cup+C=\left\{0^{2 n+1}: 0^{n} \in B\right\} \cup\left\{0^{2(m(B)+n)}: 0^{n} \in C\right\} .
$$

In $B \cup C$, all the elements from $B$ have odd length and are shorter than the elements from $C$, which have even length. We define inverses of the $\cup+$ operator:

Notation 4.6. For every $m \geq 0$ and language $A$, let

$$
\begin{aligned}
\xi(A) & :=\left\{0^{n}: n \geq 0 \wedge 0^{2 n+1} \in A\right\}, \\
\pi(m ; A) & :=\left\{0^{n}: n \geq 0 \wedge 0^{2(m+n)} \in A\right\} .
\end{aligned}
$$

If $B, C \subseteq 0^{*}$ and $B$ is finite, then $B=\xi(B \cup+C)$ and $C=\pi(m(B) ; B \cup+C)$. 
Notation 4.7. For languages $A, B \subseteq \Sigma^{*}$, we write $A \subseteq \subseteq^{*} B$ to mean that $A-B$ is finite. We write $A={ }^{*} B$ to mean that $A \subseteq{ }^{*} B$ and $B \subseteq \subseteq^{*} A$ (equivalently, the symmetric difference $A \triangle B$ is finite).

The following family of languages will be used in several places:

Definition 4.8. For all $i$, let $R_{i}$ be the language $\left(0^{*} 1^{*}\right)^{i}$.

Note that $R_{1} \subseteq R_{2} \subseteq R_{3} \subseteq \cdots$, but $R_{i+1} \nsubseteq^{*} R_{i}$ for any $i \geq 1$. Also note that $\operatorname{SUBSEQ}\left(R_{i}\right)=R_{i}$ for all $i \geq 1$.

\subsection{Definitions about subsequences.}

Notation 4.9. Given a language $A$, we call the unique minimum set $S$ satisfying

$$
\operatorname{SUBSEQ}(A)=\left\{x \in \Sigma^{*}:(\forall z \in S)[z \npreceq x]\right\}
$$

(see Equation (1)) the obstruction set of $A$ and denote it by $\operatorname{os}(A)$. In this case, we also say that $S$ obstructs $A$.

The following facts are obvious:

- The $\preceq$ relation is computable.

- For every string $x$ there are finitely many $y \preceq x$, and given $x$ one can compute a canonical index (see Notation 4.3) for the set of all such $y$.

- By various facts from automata theory, including the Myhill-Nerode minimization theorem: given a DFA, NFA, or regular expression for a language $A$, one can effectively compute the unique minimum state DFA recognizing $A$. (The minimum state DFA is given in some canonical form.)

- Given DFAs $F$ and $G$, one can effectively compute the number of states of $F$, as well as DFAs for $\overline{L(F)}, L(F) \cup L(G), L(F) \cap L(G), L(F)-L(G)$, and $L(F) \triangle L(G)$. One can also effectively determine whether or not $L(F)=\emptyset$ and whether or not $L(F)$ is finite. If $L(F)$ is finite, then one can effectively find a canonical index for $L(F)$.

- For any language $A$, the set $\operatorname{SUBSEQ}(A)$ is completely determined by $\operatorname{os}(A)$, and in fact, os $(A)=\operatorname{os}(\operatorname{SUBSEQ}(A))$.

- The strings in the obstruction set of a language must be pairwise $\preceq$ incomparable (i.e., the obstruction set is an $\preceq$-antichain). Conversely, any $\preceq$-antichain obstructs some language.

Definition 4.10. A language $A \subseteq \Sigma^{*}$ is $\preceq$-closed if $\operatorname{SUBSEQ}(A)=A$.

OBservation 4.11. A language $A$ is $\preceq$-closed if and only if there exists a language $B$ such that $A=\operatorname{SUBSEQ}(B)$.

Observation 4.12. Any infinite $\preceq$-closed set contains strings of every length.

Notation 4.13. Suppose $S \subseteq \Sigma^{*}$. We define a kind of inverse to the os $(\cdot)$ operator:

$$
\operatorname{ObsBy}(S):=\left\{x \in \Sigma^{*}:(\forall z \in S)[z \npreceq x]\right\} .
$$

Note that $\operatorname{ObsBy}(S)$ is $\preceq$-closed, and further, $\operatorname{os}(\operatorname{ObsBy}(S)) \subseteq S$ with equality holding iff $S$ is an $\preceq$-antichain. $\operatorname{ObsBy}(S)$ is the unique $\preceq$-closed set obstructed by $S$. 
Observation 4.14. For any $A, B \subseteq \Sigma^{*}, \operatorname{SuBSEQ}(A)=\operatorname{ObsBy}(B)$ if and only if $\operatorname{os}(A) \subseteq B \subseteq \overline{\operatorname{SUBSEQ}(A)}$.

The next proposition implies that finding os $(A)$ is computationally equivalent to finding a DFA for $\operatorname{SUBSEQ}(A)$.

Proposition 4.15. The following tasks are computable:

1. Given a DFA $F$, find a DFA $G$ such that $L(G)=\operatorname{SUBSEQ}(L(F))$.

2. Given the canonical index of a finite language $S \subseteq \Sigma^{*}$, compute a regular expression for (and hence the minimum-state $D F A$ recognizing) $\operatorname{ObsBy}(S)$.

3. Given a DFA $F$, decide whether or not $L(F)$ is $\preceq$-closed.

4. Given a DFA $F$, compute the canonical index of $\operatorname{os}(L(F))$.

Proof. We prove the fourth item and leave the first three as exercises for the reader.

Given DFA $F$, first compute the DFA $G$ of Item 1, above. Since os $(A)=$ $\operatorname{os}(\operatorname{SUBSEQ}(A))$ for all languages $A$, it suffices to find $\operatorname{os}(L(G))$.

Suppose that $G$ has $n$ states.

We claim that every element of $\operatorname{os}(L(G))$ has length less than $n$. Assume otherwise, i.e., that there is some string $w \in \operatorname{os}(L(G))$ with $|w| \geq n$. Then $w \notin$ $L(G)$, and as in the proof of the Pumping Lemma, there are strings $x, y, z \in \Sigma^{*}$ such that $w=x y z,|y|>0$, and $x y^{i} z \notin L(G)$ for all $i \geq 0$. In particular, $x z \notin L(G)$. But $x z \preceq w$ and $x z \neq w$, which contradicts the assumption that $w$ was a $\preceq$-minimal string in $\overline{L(G)}$. This establishes the claim.

By the claim, in order to find os $(L(G))$, we just need to check each string of length less than $n$ to see whether it is a $\preceq$-minimal string rejected by $G$.

4.3. First results. In this section we give some easy, "warm-up" proofs of membership in SUBSEQ-EX.

Notation 4.16. $\mathcal{F}$ is the set of all finite sets of strings.

Proposition 4.17. $\mathcal{F} \in$ SUBSEQ-EX.

Proof. Let $M$ be a learner that, when $A \in \mathcal{F}$ is on the tape, outputs $k_{1}, k_{2}, \ldots$, where each $k_{i}$ is an index of a DFA that recognizes $\operatorname{SUBSEQ}\left(A \cap \Sigma^{\leq i}\right)$. Clearly, $M$ learns $\operatorname{SUBSEQ}(A)$.

More generally, we have

Proposition 4.18. REG $\in$ SUBSEQ-EX.

Proof. When $A$ is on the tape, for $n=0,1,2, \ldots$, the learner $M$

1. finds the least $k$ such that $A \cap \Sigma^{<n}=L\left(F_{k}\right) \cap \Sigma^{<n}$, then

2. outputs the $\ell$ such that $L\left(F_{\ell}\right)=\operatorname{SUBSEQ}\left(L\left(F_{k}\right)\right)$ (see Proposition 4.15(1)).

If $A$ is regular, then clearly $M$ will converge to the least $k$ such that $A=L\left(F_{k}\right)$, whence $M$ will converge to the least $\ell$ such that $L\left(F_{\ell}\right)=\operatorname{SUBSEQ}(A)$.

Even more generally, we have

Proposition 4.19. CFL $\in$ SUBSEQ-EX, where CFL is the class of all context-free languages. 
Proof. Similarly to the proof of Proposition 4.18, when $A$ is on the tape, for $n=0,1,2, \ldots$, the learner $M$ initially finds the first context-free grammar $G$ (in some standard ordering) such that $A \cap \Sigma^{<n}=L(G) \cap \Sigma^{<n}$. By a result of van Leeuwen [34] (alternatively, see [11]), given a context-free grammar for a language $A$, one can effectively find a DFA for $\operatorname{SUBSEQ}(A)$. The learner $M$ thus computes and outputs the index of a DFA recognizing $\operatorname{SUBSEQ}(L(G))$. $\dashv$

We show later (Corollary 5.7) that coCFL $\notin$ SUBSEQ-EX, where coCFL is the class of complements of context-free languages.

4.4. Variants on SUBSEQ-EX. In this section, we note some obvious inclusions among the variant notions of SUBSEQ-EX. We also define relativized SUBSEQ-EX.

Obviously,

(2) SUBSEQ-EX $_{0} \subseteq$ SUBSEQ-EX ${ }_{1} \subseteq$ SUBSEQ-EX $_{2} \subseteq \cdots \subseteq$ SUBSEQ-EX.

We will extend this definition into the transfinite later. Clearly,

(3) SUBSEQ-EX $=$ SUBSEQ-EX ${ }^{0} \subseteq$ SUBSEQ-EX $^{1} \subseteq \cdots \subseteq$ SUBSEQ-EX $*$.

Finally, it is evident that $[a, b]$ SUBSEQ-EX $\subseteq[c, d]$ SUBSEQ-EX if $a \geq c$ and $b \leq d$.

Definition 4.20. If $X \subseteq \mathbb{N}$, then SUBSEQ-EX ${ }^{X}$ is the same as SUBSEQ-EX except that we allow the learner to be an oracle TM using oracle $X$.

We may combine these variants in a large variety of ways.

\section{S5. Main results.}

5.1. Standard learning. We start by giving an example of something in SUBSEQ-EX that contains non-context-free languages. We will give more extreme examples in Section 6.

Definition 5.1. For all $i \in \mathbb{N}$, let

$$
\mathcal{S}_{i}:=\left\{A \subseteq \Sigma^{*}:|\operatorname{os}(A)|=i\right\}
$$

Also let

$$
\mathcal{S}_{\leq i}:=\mathcal{S}_{0} \cup \mathcal{S}_{1} \cup \cdots \cup \mathcal{S}_{i}=\left\{A \subseteq \Sigma^{*}:|\operatorname{os}(A)| \leq i\right\}
$$

Note that each $\mathcal{S}_{i}$ contains languages of arbitrary complexity. For example, if $\Sigma=\left\{a_{1}, \ldots, a_{k}\right\}$, then $\mathcal{S}_{0}$ contains (among others) all languages $A$ such that $A \cap\left(a_{1} \cdots a_{k}\right)^{*}$ is infinite.

Proposition 5.2. $\mathcal{S}_{i} \in$ SUBSEQ-EX for all $i \in \mathbb{N}$. In fact, there is a computable function $\ell$ such that for each $i, M_{\ell(i)}$ learns $\operatorname{SUBSEQ}(A)$ for every $A \in \mathcal{S}_{i}$.

Proof. Given $A$ on its tape, let $M=M_{\ell(i)}$ behave as follows, for $n=$ $0,1,2, \ldots$ :

1. Compute $N=\operatorname{os}\left(A \cap \Sigma^{\leq n}\right) \cap \Sigma^{\leq n}$.

2. If $|N|<i$, then go on to the next $n$.

3. Let $x_{1}, \ldots, x_{i}$ be the $i$ shortest strings in $N$. If there is a tie, i.e., if there is more than one set of $i$ shortest strings in $N$, then go on to the next $n$. 
4. Output the index of the DFA recognizing $\operatorname{ObsBy}\left(\left\{x_{1}, \ldots, x_{i}\right\}\right)$. It is easy to see that $\left\{x_{1}, \ldots, x_{i}\right\}$ converges to $\operatorname{os}(A)$ in the limit.

Remark. A consequence of Proposition 5.2 is that learning just the cardinality of $\operatorname{os}(A)$ is equivalent to learning (a DFA for) $\operatorname{SUBSEQ}(A)$ : Suppose we are given a learner $M$ that with $A$ on its tape outputs natural numbers $i_{1}, i_{2}, \ldots$ With $A$ on the tape, for $n=1,2, \ldots$, run $M_{\ell\left(i_{n}\right)}$ on $A$ for $n$ steps and output its most recent output (if there is one). If $M$ 's outputs converge to $|\operatorname{os}(A)|$, then clearly we learn $\operatorname{SUBSEQ}(A)$ this way.

It was essentially shown in [11] that DEC $\notin$ SUBSEQ-EX. The proof there can be tweaked to show the stronger result that $\mathrm{P} \notin \mathrm{SUBSEQ}-\mathrm{EX}$. We include the stronger result here.

ThEOREM 5.3 ([11]). P $\notin$ SUBSEQ-EX. In fact, there is a computable function $g$ such that for all $e$, setting $A=L\left(P_{g(e)}\right)$, we have $A \subseteq 0^{*}$ and $\operatorname{SUBSEQ}(A)$ is not learned by $M_{e}$.

Proof. Assume, by way of contradiction, that $\mathrm{P} \in \mathrm{SUBSEQ}-\mathrm{EX}$ via $M_{e}$. Then we effectively construct a machine $N_{e}$ that implements the following recursive polynomial-time algorithm for computing $A$. Let $j_{0}$ be the unique index such that $L\left(F_{j_{0}}\right)=0^{*}$.

On input $x$ :

1. If $x \notin 0^{*}$ then reject. (This will ensure that $A \subseteq 0^{*}$.)

2. Let $x=0^{n}$. Using no more than $n$ computational steps, recursively run $N_{e}$ on inputs $\varepsilon, 0,00, \ldots, 0^{\ell_{n}-1}$ to compute $A(\varepsilon), A(0), A(00), \ldots, A\left(0^{\ell_{n}-1}\right)$, where $\ell_{n} \leq n$ is largest such that this can all be done within $n$ steps. Set $S_{n}:=A \cap 0^{<\ell_{n}}$.

3. Simulate $M_{e}$ for $\ell_{n}-1$ steps with $S_{n}$ on its tape. If $M_{e}$ does not output anything within this time, then reject. [Note that $M_{e}$ only has time to scan its tape on cells corresponding to inputs $\varepsilon, 0,00, \ldots, 0^{\ell_{n}-1}$ (and perhaps some inputs not in $\left.0^{*}\right)$.]

4. Let $k$ be the most recent index output by $M_{e}$ within $\ell_{n}-1$ steps with $S_{n}$ on its tape.

5. If $k=j_{0}$ (i.e., if $L\left(F_{k}\right)=0^{*}$ ), then reject; else accept.

This algorithm runs in polynomial time for each fixed $e$, and thus $A=L\left(N_{e}\right) \in$ P. Further, given $e$ we can effectively compute an index $i$ such that $A=L\left(P_{i}\right)$. We let $g(e)=i$.

We note the following:

- It is clear that the sequence $\ell_{0}, \ell_{1}, \ell_{2}, \ldots$ is monotone and unbounded.

- When $M_{e}$ is simulated in step 3 , it behaves the same way with $S_{n}$ on its tape as with $A$ on its tape, because it does not run long enough to examine any place on the tape where $S_{n}$ and $A$ may differ.

We now show that $M_{e}$ does not learn $\operatorname{SUBSEQ}(A)$. Assume otherwise, and let $k_{1}, k_{2}, \ldots$ be the sequence of outputs of $M_{e}$ with $A$ on the tape. By assumption, there is a $k^{\prime}=\lim _{n \rightarrow \infty} k_{n}$ such that $L\left(F_{k^{\prime}}\right)=\operatorname{SUBSEQ}(A)$. If $L\left(F_{k^{\prime}}\right)=0^{*}$, then for all large enough $n$, the algorithm rejects $0^{n}$ in Step 5 , making $A$ finite, which makes $\operatorname{SUBSEQ}(A)$ finite. If $L\left(F_{k^{\prime}}\right) \neq 0^{*}$, then the algorithm accepts $0^{n}$ in 
Step 5 for all large enough $n$, making $A$ infinite, which makes $\operatorname{SUBSEQ}(A)=0^{*}$. In either case, $L\left(F_{k^{\prime}}\right) \neq \operatorname{SUBSEQ}(A)$; a contradiction.

Remark. The fact that $0^{*}$ has a unique index $j_{0}$ allows for an easy equality test of indices in Step 5 above, and this is crucial to keeping the algorithm and its polynomial-time efficiency proof simple. Had we used the more naïve test " $L\left(F_{k}\right)=0^{*}$ " instead without unique indices, we would either need to make the additional - and nontrivial - assumption that our indices encode the structure of the DFAs in an efficient way (for example, if we restrict ourselves to minimum DFAs indexed in lexicographically increasing order, it is not at all clear that we can determine the structure of the $k$ th minimum DFA (and hence decide whether it recognizes $0^{*}$ ) in time polynomial in $\log _{2} k$ ), or else have to build in an additional "wait-and-see" delay in deciding acceptance or rejection until our input $x$ was large enough to have the time to decide whether $L\left(F_{k}\right)=0^{*}$ for one of $M_{e}$ 's previous hypotheses $k$. This would be especially important if we allowed BC-style learning for $M_{e}$. Having unique indices for regular languages frees us from having to make any such complications.

\section{Corollary 5.4. $\mathrm{P} \cap \mathcal{P}\left(0^{*}\right) \notin$ SUBSEQ-EX.}

The nonmembership of $\mathrm{P}$ in SUBSEQ-EX is an instance of a general negative result-Theorem 5.6 below. The following concepts are reasonably standard.

Definition 5.5. We say that a function $g: \mathbb{N}^{+} \rightarrow \mathbb{N}^{+}$is a subrecursive programming system (SPS) if $g$ is computable and $M_{g(e)}$ is total for all $e$. We also define

- $\mathcal{L}(g):=\left\{L\left(M_{g(e)}\right): e \in \mathbb{N}^{+}\right\}$, and

- $\mathrm{FIN}_{g}:=\left\{e: L\left(M_{g(e)}\right)\right.$ is finite $\}$.

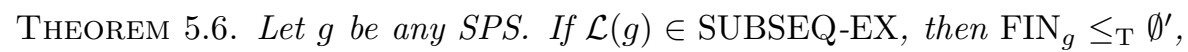
where $\emptyset^{\prime}$ is the halting problem.

Proof. Suppose that $\mathcal{L}(g) \in$ SUBSEQ-EX witnessed by a learner $N$. Define $f(e, n)$ to be the following computable function:

On input $e, n \in \mathbb{N}^{+}$,

1. Simulate $N$ with $L\left(M_{g(e)}\right)$ on its tape, and let $k_{n}$ be its $n$th output.

2. If $L\left(F_{k_{n}}\right)$ is finite, then let $f(e, n)=1$; else let $f(e, n)=0$.

Clearly,

$$
e \in \mathrm{FIN}_{g} \Longleftrightarrow L\left(M_{g(e)}\right) \text { is finite } \Longleftrightarrow \operatorname{SUBSEQ}\left(L\left(M_{g(e)}\right)\right) \text { is finite. }
$$

Since $L\left(F_{k_{n}}\right)=\operatorname{SUBSEQ}\left(L\left(M_{g(e)}\right)\right)$ for cofinitely many $n$, we have $\operatorname{FIN}_{g}(e)=$ $\lim _{n} f(e, n)$, and hence by the Limit Lemma (see [33]), $\mathrm{FIN}_{g} \leq_{\mathrm{T}} \emptyset^{\prime}$.

The following fact stands in sharp contrast to Proposition 4.19.

Corollary 5.7. coCFL $\notin$ SUBSEQ-EX, where coCFL is the class of all complements of context-free languages.

Proof. The cofiniteness problem for context-free grammars is known to be $\Sigma_{2}$-complete [21], and thus not computable in $\emptyset^{\prime}$.

We can learn more with oracle access to the halting problem. 


\section{TheOrem 5.8. CE $\in$ SUBSEQ-EX $\emptyset^{\emptyset^{\prime}}$.}

Proof. Consider a learner $M$ for all c.e. languages that behaves as follows: When the characteristic sequence of a c.e. language $A$ is on the tape, $M$ learns (with the help of $\emptyset^{\prime}$ ) a c.e. index for $A$ by finding, for each $n=0,1,2, \ldots$, the least $e$ such that $W_{e} \cap \Sigma^{\leq n}=A \cap \Sigma^{\leq n}$. Eventually $M$ will settle on a correct $e$, assuming $A$ is c.e. Let $e_{n}$ be the $n$th index found by $M$. Upon finding $e_{n}, M$ uses $\emptyset^{\prime}$ to determine, for each $w \in \Sigma \leq n$, whether or not there is a $z \in W_{e_{n}}$ such that $w \preceq z . M$ collects the set $D$ of all $w \in \Sigma^{\leq n}$ for which this is not the case, then outputs (an index for) the DFA recognizing $\operatorname{ObsBy}(D)$ as in Proposition 4.15(2).

For all large enough $n$ we have $A=W_{e_{n}}$, and all strings in $\operatorname{os}(A)$ will have length at most $n$. Thus $M$ eventually outputs a DFA for $\operatorname{SUBSEQ}(A)$.

5.2. Alternate learning modes. Our main focus is on SUBSEQ-EX and its variants, but in this section we digress briefly to consider three alternatives to $\operatorname{SUBSEQ-EX~for~learning~} \operatorname{SUBSEQ}(A)$ :

1. BC ("behaviorally correct") learning $[4,5,7]$,

2. inferring devices for $\operatorname{SUBSEQ}(A)$ other than DFAs, and

3. learning $\operatorname{SUBSEQ}(A)$ given one-sided data (positive or negative examples). This subsection is not needed for the rest of the paper.

5.2.1. BC learning of $\operatorname{SUBSEQ}(A)$. For every regular language there is a unique index for a DFA that recognizes it (Notation 4.3(2)). Thus in the context of standard, anomaly-free learning, there is no difference between the EXand BC-styles of learning $\operatorname{SUBSEQ}(A)$. Even when learning other devices or learning with anomalies, we show that the EX and BC identification criteria for $\operatorname{SUBSEQ}(A)$ are still largely equivalent (Propositions 5.9 and 5.19, below, respectively).

5.2.2. Learning other devices for $\operatorname{SUBSEQ}(A)$. Learning $\operatorname{SUBSEQ}(A)$ turns out to be largely independent of the type of device the learner must output.

Proposition 5.9. Let $\mathcal{A}$ be a class of languages. $\mathcal{A} \in$ SUBSEQ-EX if and only if there is a learner $M$ that, with any $A \in \mathcal{A}$ on its tape, cofinitely often outputs a co-c.e. index for $\operatorname{SUBSEQ}(A)$ (i.e., an e such that $\left.W_{e}=\overline{\operatorname{SUBSEQ}(A)}\right)$.

Proof. The forward implication is obvious. For the reverse implication, we are given a learner $M$ that with $A \in \mathcal{A}$ on its tape outputs indices $e_{1}, e_{2}, \ldots$ such that $W_{e_{j}}=\overline{\operatorname{SUBSEQ}(A)}$ for all but finitely many $j$. We learn a DFA for $\operatorname{SUBSEQ}(A)$ as follows:

With $A$ on the tape, for $n=1,2, \ldots$ :

1. Simulate $M$ on $A$ to find $e_{1}, \ldots, e_{n}$.

2. Find $\operatorname{WRONG}(n):=\left\{j: 1 \leq j \leq n \wedge W_{e_{j}, n} \cap \operatorname{SUBSEQ}\left(A \cap \Sigma^{<n}\right) \neq \emptyset\right\}$.

3. Output the index of a DFA for $\operatorname{ObsBy}\left(S_{n}\right)$ (Proposition 4.15(2)), where

$$
S_{n}:=\bigcup\left\{W_{e_{j}, n}: j \in\{1, \ldots, n\}-\operatorname{WRONG}(n)\right\} .
$$

If $W_{e_{j}} \cap \operatorname{SUBSEQ}(A) \neq \emptyset$, then evidently $j \in \operatorname{WRONG}(n)$ for cofinitely many $n$. Since there are only finitely many such $j$, we have that $S_{n} \subseteq \overline{\operatorname{SUBSEQ}(A)}$ for cofinitely many $n$. Finally, os $(A) \subseteq S_{n} \subseteq \overline{\operatorname{SUBSEQ}(A)}$ for cofinitely many $n$, 
because $\operatorname{os}(A)$ is finite and $\operatorname{os}(A) \subseteq W_{e_{j}}=\overline{\operatorname{SUBSEQ}(A)}$ for some $j$. For all such $n, \operatorname{SUBSEQ}(A)=\operatorname{ObsBy}\left(S_{n}\right)$ by Observation 4.14 .

Proposition 5.9 obviously applies to any devices from which equivalent co-c.e. indices can be found effectively, e.g., polynomial-time TMs, context-sensitive grammars, total TMs, etc. This result contrasts with results in [11], where it was shown, for example, that one cannot effectively convert a polynomial-time TM deciding $A$ into a total TM deciding $\operatorname{SUBSEQ}(A)$.

Proposition 5.9 does not extend to learning a c.e. index for SUBSEQ $(A)$. This follows easily from a standard result in inductive inference that goes back at least to Gold [20], and whose proof is analogous to that of Proposition 4.19. The corollary that follows should be compared with Theorem 5.6.

Proposition 5.10 (Gold [20]). Let $g$ be an SPS (see Definition 5.5). Then there is a learner $M$ that, given any language $A \in \mathcal{L}(g)$ on its tape, converges to an index e such that $A=L\left(M_{g(e)}\right)$.

Corollary 5.11. For any SPS $g$ there is a learner $M$ that given any $A \in \mathcal{L}(g)$ on its tape, converges to a c.e. index for $\operatorname{SUBSEQ}(A)$.

Proof. This follows from Proposition 5.10 and the easy fact, proved in [11], that one can compute a c.e. index for $\operatorname{SUBSEQ}(A)$ given a c.e. index for $A$. $\dashv$

5.2.3. Learning $\operatorname{SUBSEQ}(A)$ from one-sided data. When a learner learns from positive examples, rather than having the characteristic function of $A$ on its tape, the learner is instead given an infinite list of all the strings in $A$ in arbitrary order [2]. Repetitions are allowed in the list, as well as any number of occurrences of the special symbol ' $*$ ' meaning "no data." Such a list is called a text for $A$. Learning from negative examples is similar except that the learner is given a text for $\bar{A}$. A class $\mathcal{A}$ of c.e. languages is in TxtEX iff there is a learner $M$ that, given any text for any $A \in \mathcal{A}$, converges to a c.e. index for $A$. Variants of TxtEX have been extensively studied $[24,1]$. Here we give some brief observations about learning $\operatorname{SUBSEQ}(A)$ from one-sided data (either positive or negative examples), which may serve as a basis for further research.

Definition 5.12. A class $\mathcal{A}$ of languages is SUBSEQ-learnable from positive (respectively negative) data if there is a learner $M$ that, given any text for any $A \in \mathcal{A}$ (respectively, text for $\bar{A}$ ), converges on a $\operatorname{DFA}$ for $\operatorname{SUBSEQ}(A)$.

ObSERVATIOn 5.13. If $\mathcal{A}$ is SUBSEQ-learnable from either positive or negative data, then $\mathcal{A} \in \mathrm{SUBSEQ}-\mathrm{EX}$.

Given text for $A$, it is easy to generate text for $\operatorname{SUBSEQ}(A)$, thus we have:

Observation 5.14. For any class $\mathcal{A}$ of languages, if $\{\operatorname{SUBSEQ}(A): A \in \mathcal{A}\}$ is SUBSEQ-learnable from positive data, then so is $\mathcal{A}$ (and thus $\mathcal{A} \in \mathrm{SUBSEQ}-\mathrm{EX}$ ). It follows, for example, that $\left\{0^{*}\right\} \cup\left\{0^{<n}: n \in \mathbb{N}\right\}$ is not SUBSEQ-learnable from positive data (see Corollary 5.4).

The converse of Observation 5.14 is false, witnessed by the class

$$
\left\{\left\{0^{|A|+1}\right\} \cup+A: A \subseteq 0^{*} \text { is finite }\right\} \cup\left\{\{\varepsilon\} \cup+A: A \subseteq 0^{*} \text { is infinite }\right\},
$$

which is SUBSEQ-learnable from positive data (without mind-changes).

The proof of Proposition 5.9 yields the next observation. 
OBSERVATION 5.15. The class of $\preceq$-closed languages is SUBSEQ-learnable from negative data.

Our last observation regards learning a c.e. index for $\operatorname{SUBSEQ}(A)$. The proof is similar to that of Corollary 5.11.

Observation 5.16. If $\mathcal{A} \in \mathrm{TxtEX}$, then there is a learner $M$ that, given any text for any $A \in \mathcal{A}$, converges on a c.e. index for $\operatorname{SUBSEQ}(A)$.

5.3. Anomalies. The next theorem shows that the anomalies hierarchy of Equation (3) collapses completely. In other words, allowing the output DFA to be wrong on (say) five places does not increase learning power.

Theorem 5.17. SUBSEQ-EX = SUBSEQ-EX*. In fact, there is a computable $h$ such that for all $e$ and languages $A$, if $M_{e}$ learns $\operatorname{SUBSEQ}(A)$ with finitely many anomalies, then $M_{h(e)}$ learns $\operatorname{SUBSEQ}(A)$ (with zero anomalies).

Proof. Given $e$, we let $M_{h(e)}$ learn $\operatorname{SUBSEQ}(A)$ by finding better and better approximations to it: For increasing $n, M_{h(e)}$ with $A$ on its tape approximates $\operatorname{SUBSEQ}(A)$ by examining its tape directly on strings in $\Sigma^{<n}$ (where there could be anomalies) and relying on $L(F)$ for strings of length $\geq n$, where $F$ is the most recent output of $M_{e}$. Here is the algorithm for $M_{h(e)}$ :

When language $A$ is on the tape:

1. Run $M_{e}$ with $A$. Wait for $M_{e}$ to output something.

2. Whenever $M_{e}$ outputs some hypothesis $k$, do the following:

(a) Let $n$ be the number of times $M_{e}$ has output a hypothesis thus far (thus $k$ is $M_{e}$ 's $n$th hypothesis).

(b) Compute a DFA $G$ recognizing $\operatorname{SUBSEQ}\left(\left(A \cap \Sigma^{<n}\right) \cup\left(L\left(F_{k}\right) \cap \Sigma^{\geq n}\right)\right)$.

(c) Output the index of $G$.

If $M_{e}$ learns $\operatorname{SUBSEQ}(A)$ with finite anomalies, then there is a DFA $F$ such that, for all large enough $n, M_{e}$ outputs an index for $F$ as its $n$th hypothesis, and furthermore $L(F) \triangle \operatorname{SUBSEQ}(A) \subseteq \Sigma^{<n}$, that is, all anomalies are of length less than $n$. For any such $n$, let $G_{n}$ be the DFA output by $M_{h(e)}$ after the $n$th hypothesis of $M_{e}$. We have

$$
\begin{aligned}
L\left(G_{n}\right) & =\operatorname{SUBSEQ}\left(\left(A \cap \Sigma^{<n}\right) \cup\left(L(F) \cap \Sigma^{\geq n}\right)\right) \\
& =\operatorname{SUBSEQ}\left(\left(A \cap \Sigma^{<n}\right) \cup\left(\operatorname{SUBSEQ}(A) \cap \Sigma^{\geq n}\right)\right) \\
& =\operatorname{SUBSEQ}(A) .
\end{aligned}
$$

Thus $M_{h(e)}$ learns $\operatorname{SUBSEQ}(A)$.

One could define a looser notion of learning with finite anomalies: The learner is only required to eventually (i.e., cofinitely often) output indices for DFAs whose languages differ a finite amount from $\operatorname{SUBSEQ}(A)$, but these languages need not all be the same. This is reminiscent of the $\mathrm{BC}$ criterion of inductive inference $[4,5,7]$.

Definition 5.18. For a learner $M$ and language $A$, say that $M$ weakly learns $\operatorname{SUBSEQ}(A)$ with finite anomalies if, when $A$ is on the tape, $M$ outputs an infinite sequence $k_{1}, k_{2}, \ldots$ such that $\operatorname{SUBSEQ}(A)={ }^{*} L\left(F_{k_{i}}\right)$ for all but finitely many $i$. 
A class $\mathcal{C}$ of languages is in SUBSEQ-BC* if there is a learner $M$ that, for every $A \in \mathcal{C}$, weakly learns $\operatorname{SUBSEQ}(A)$ with finite anomalies.

Clearly, SUBSEQ-EX* $\subseteq$ SUBSEQ-BC* . We use Theorem 5.17 to get an even stronger collapse.

Proposition 5.19. SUBSEQ-EX = SUBSEQ-BC* . In fact, there is a computable function $b$ such that for all $e$ and $A$, if $M_{e}$ weakly learns $A$ with finite anomalies, then $M_{b(e)}$ learns $A$ (without anomalies).

Proof. Let $c$ be a computable function such that for all $e$ and $A, M_{c(e)}$ with $A$ on the tape simulates $M_{e}$ with $A$ on the tape, and (supposing $M_{e}$ outputs $\left.k_{1}, k_{2}, \ldots\right)$ whenever $M_{e}$ outputs $k_{n}, M_{c(e)}$ finds the least $j \leq n$ such that $L\left(F_{k_{j}}\right)={ }^{*} L\left(F_{k_{n}}\right)$, and outputs $k_{j}$ instead. (Such a $j$ can be computed.)

Now suppose $M_{e}$ weakly learns $\operatorname{SUBSEQ}(A)$ with finite anomalies, and let $k_{1}, k_{2}, \ldots$ be the outputs of $M_{e}$ with $A$ on the tape. Let $j$ be least such that $L\left(F_{k_{j}}\right)={ }^{*} \operatorname{SUBSEQ}(A)$. Then for cofinitely many $n$, we have $L\left(F_{k_{n}}\right)={ }^{*}$ $\operatorname{SUBSEQ}(A)$, and so $L\left(F_{k_{n}}\right)=^{*} L\left(F_{k_{j}}\right)$ as well, but $L\left(F_{k_{n}}\right) \neq^{*} L\left(F_{k_{\ell}}\right)$ for all $\ell<j$. Thus $M_{c(e)}$ outputs $k_{j}$ cofinitely often, and so $M_{c(e)}$ learns $\operatorname{SUBSEQ}(A)$ with finite anomalies (not weakly!).

Now we let $b=h \circ c$, where $h$ is the function of Theorem 5.17. If $M_{e}$ weakly learns $\operatorname{SUBSEQ}(A)$ with finite anomalies, then $M_{c(e)}$ learns $\operatorname{SUBSEQ}(A)$ with finite anomalies, and so $M_{b(e)}=M_{h(c(e))}$ learns $\operatorname{SUBSEQ}(A)$.

5.4. Mind-changes. The next theorems show that the mind-change hierarchy of Equation (2) separates. In other words, if you allow more mind-changes then you give the learning device more power.

Definition 5.20. For every $i>0$, define the class

$$
\mathcal{C}_{i}=\left\{A \subseteq 0^{*}:|A| \leq i\right\}
$$

Proposition 5.21. $\mathcal{C}_{i} \in \mathrm{SUBSEQ}_{\mathrm{EX}}$ for all $i \in \mathbb{N}$. In fact, there is a single learner $M$ that for each $i$ learns $\operatorname{SUBSEQ}(A)$ for every $A \in \mathcal{C}_{i}$ with at most $i$ mind-changes.

Proof. Let $M$ be as in the proof of Proposition 4.17. Clearly, $M$ learns any $A \in \mathcal{C}_{i}$ with at most $|A|$ mind-changes.

TheOrem 5.22. For each $i>0, \mathcal{C}_{i} \notin$ SUBSEQ-EX $_{i-1}$. In fact, there is a computable function $\ell$ such that, for each e and $i>0, M_{\ell(e, i)}$ is total and decides a unary language $A_{e, i}=L\left(M_{\ell(e, i)}\right) \subseteq 0^{*}$ such that $\left|A_{e, i}\right| \leq i$ and $M_{e}$ does not learn $\operatorname{SUBSEQ}\left(A_{e, i}\right)$ with fewer than $i$ mind-changes.

Proof. Given $e$ and $i>0$ we construct a machine $N=M_{\ell(e, i)}$ that implements the following recursive algorithm to compute $A_{e, i}$ :

Given input $x$,

1. If $x \notin 0^{*}$, then reject. (This ensures that $A_{e, i} \subseteq 0^{*}$.) Otherwise, let $x=0^{n}$.

2. Recursively compute $S_{n}=A_{e, i} \cap 0^{<n}$.

3. Simulate $M_{e}$ for $n-1$ steps with $S_{n}$ on the tape. (Note that $M_{e}$ does not have time to read any of the tape corresponding to inputs $0^{n^{\prime}}$ for $n^{\prime} \geq n$.) If $M_{e}$ does not output anything within this time, then reject. 
4. Let $k$ be the most recent output of $M_{e}$ in the previous step, and let $c$ be the number of mind-changes that $M_{e}$ has made up to this point. If $c<i$ and $L\left(F_{k}\right)=\operatorname{SUBSEQ}\left(S_{n}\right)$, then accept; else reject.

In step 3 of the algorithm, $M_{e}$ behaves the same with $S_{n}$ on its tape as it would with $A_{e, i}$ on its tape, given the limit on its running time.

Let $A_{e, i}=\left\{0^{z_{0}}, 0^{z_{1}}, \ldots\right\}$, where $z_{0}<z_{1}<\cdots$ are natural numbers.

Claim. For $0 \leq j$, if $z_{j}$ exists, then $M_{e}$ (with $A_{e, i}$ on its tape) must output a DFA for $\operatorname{SUBSEQ}\left(S_{z_{j}}\right)$ within $z_{j}-1$ steps, having changed its mind at least $j$ times when this occurs.

Proof of The ClAim. We proceed by induction on $j$ : For $j=0$, the string $0^{z_{0}}$ is accepted by $N$ only if within $z_{0}-1$ steps $M_{e}$ outputs a $k$ where $L\left(F_{k}\right)=$ $\emptyset=\operatorname{SUBSEQ}\left(S_{z_{0}}\right)$; no mind-changes are required. Now assume that $j \geq 0$ and $z_{j+1}$ exists, and also (for the inductive hypothesis) that within $z_{j}-1$ steps $M_{e}$ outputs a DFA for $\operatorname{SUBSEQ}\left(S_{z_{j}}\right)$ after at least $j$ mind-changes. We have $S_{z_{j}} \subseteq 0^{<z_{j}}$ but $0^{z_{j}} \in S_{z_{j+1}}$, and so $\operatorname{SUBSEQ}\left(S_{z_{j}}\right) \neq \operatorname{SUBSEQ}\left(S_{z_{j+1}}\right)$. Since $N$ accepts $0^{z_{j+1}}$, it must be because $M_{e}$ has just output a DFA for $\operatorname{SUBSEQ}\left(S_{z_{j+1}}\right)$ within $z_{j+1}-1$ steps, thus having changed its mind at least once since the $z_{j}$ th step of its computation, making at least $j+1$ mind-changes in all. So the claim holds for $j+1$. End of Proof of Claim

First we show that $A_{e, i} \in \mathcal{C}_{i}$. Indeed, by the Claim above, $z_{i}$ cannot exist, because the algorithm would explicitly reject such a string $0^{z_{i}}$ if $M_{e}$ made at least $i$ mind-changes in the first $z_{i}-1$ steps. Thus we have $\left|A_{e, i}\right| \leq i$, and so $A_{e, i} \in \mathcal{C}_{i}$

Next we show that $M_{e}$ cannot learn $\operatorname{SUBSEQ}\left(A_{e, i}\right)$ with fewer than $i$ mindchanges. Suppose that with $A_{e, i}$ on its tape, $M_{e}$ makes fewer than $i$ mindchanges. Suppose also that there is a $k$ output cofinitely many times by $M_{e}$. Let $t$ be least such that $t \geq m\left(A_{e, i}\right)$ and $M_{e}$ outputs $k$ within $t-1$ steps. Then $L\left(F_{k}\right) \neq \operatorname{SUBSEQ}\left(A_{e, i}\right)$, for otherwise the algorithm would accept $0^{t}$ and so $0^{t} \in A_{e, i}$, contradicting the choice of $t$. It follows that $M_{e}$ cannot learn $\operatorname{SUBSEQ}\left(A_{e, i}\right)$ with fewer than $i$ mind-changes.

5.4.1. Transfinite mind-changes and procrastination. This subsection may be skipped on first reading. We extend the results of the previous subsection into the transfinite. Freivalds \& Smith defined $\mathrm{EX}_{\alpha}$ for all constructive ordinals $\alpha$ [13]. When $\alpha<\omega$, the definition is the same as the finite mind-change case above. If $\alpha \geq \omega$, then the learner may revise its bound on the number of mind-changes during the computation. The learner may be able to revise more than once, or even compute a bound on the number of future revisions, and this bound itself could be revised, et cetera, depending on the size of $\alpha$. After giving some basic

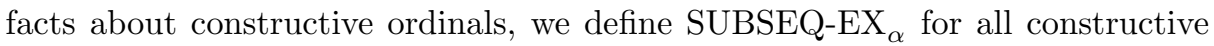
$\alpha$, then show that this transfinite hierarchy separates. Our definition is slightly different from, but equivalent to, the definition in [13]. For general background on constructive ordinals, see [30, 31].

Church defined the constructive (computable) ordinals, and Kleene defined a partially ordered set $\left\langle\mathcal{O},<_{\mathcal{O}}\right\rangle$ of notations for constructive ordinals, where $\mathcal{O} \subseteq \mathbb{N} .\left\langle\mathcal{O},<_{\mathcal{O}}\right\rangle$ may be defined as the least partial order that satisfies the following closure properties: 
- $<_{\mathcal{O}} \subseteq \mathcal{O} \times \mathcal{O}$, and $<_{\mathcal{O}}$ is transitive.

- $0 \in \mathcal{O}$.

- If $a \in \mathcal{O}$ then $2^{a} \in \mathcal{O}$ and $a<_{\mathcal{O}} 2^{a}$.

- If $M_{e}$ is total (with inputs in $\mathbb{N}$ ) and

$$
M_{e}(0)<_{\mathcal{O}} M_{e}(1)<_{\mathcal{O}} M_{e}(2)<_{\mathcal{O}} \cdots
$$

then $3 \cdot 5^{e} \in \mathcal{O}$ and $M_{e}(n)<_{\mathcal{O}} 3 \cdot 5^{e}$ for all $n \in \mathbb{N}$.

$\left\langle\mathcal{O},<_{\mathcal{O}}\right\rangle$ has the structure of a well-founded tree. For $a \in \mathcal{O}$ we let $\|a\|$ be the ordinal rank of $a$ in the partial ordering. ${ }^{4}$ Then $a$ is a notation for the ordinal $\|a\|$. An ordinal $\alpha$ is constructive if it has a notation in $\mathcal{O}$. We let $\omega_{1}^{\mathrm{CK}}$ be the set of all constructive ordinals, i.e., the height of the tree $\left\langle\mathcal{O},<_{\mathcal{O}}\right\rangle . \omega_{1}^{\mathrm{CK}}$ is itself a countable ordinal - the least nonconstructive ordinal.

It can be shown that $\left\langle\mathcal{O},<_{\mathcal{O}}\right\rangle$ has individual branches of height $\omega_{1}^{\mathrm{CK}}$. If $B \subseteq \mathcal{O}$ is such a branch, then every constructive ordinal has a unique notation in $B$. In keeping with [13], we fix a single such branch $\mathrm{ORD} \subseteq \mathbb{N}$ of unique notations once and for all, then identify (for computational purposes) each constructive ordinal with its notation in ORD. (It is likely that the classes we define depend on the actual system ORD chosen, but our results hold for any such branch that we fix.)

We note the following basic facts about constructive ordinals $\alpha<\omega_{1}^{\mathrm{CK}}$ :

- It is a computable task to determine whether $\alpha$ is zero, $\alpha$ is a successor, or $\alpha$ is a limit. $\left(\alpha=0, \alpha=2^{a}\right.$ for some $a$, or $\alpha=3 \cdot 5^{e}$ for some $e$, respectively.)

- If $\alpha$ is a successor, then its predecessor $\left(=\log _{2} \alpha\right)$ can be computed.

- If $\alpha=3 \cdot 5^{e}$ is a limit, then we can compute $M_{e}(0), M_{e}(1), M_{e}(2), \ldots$, and this is a strictly ascending sequence of ordinals with limit $\alpha$.

- We can compute the unique ordinals $\lambda$ and $n$ such that $\lambda$ is zero or a limit, $n<\omega$, and $\lambda+n=\alpha$. We denote this $n$ by $N(\alpha)$ and this $\lambda$ by $\Lambda(\alpha)$.

- There is a computably enumerable set $S$ such that for all $b \in$ ORD and $a \in \mathbb{N},(a, b) \in S$ iff $a \in$ ORD and $\|a\|<\|b\|$. That is, given an ordinal $\alpha<\omega_{1}^{\mathrm{CK}}$, we can effectively enumerate all $\beta<\alpha$, and this enumeration is uniform in $\alpha$.

- Thanks to ORD being totally ordered, the previous item implies that we can effectively determine whether or not $\alpha<\beta$ for any $\alpha, \beta<\omega_{1}^{\mathrm{CK}}$. That is, there is a partial computable predicate that extends the ordinal less-than relation on ORD.

Definition 5.23. A procrastinating learner is a learner $M$ equipped with an additional ordinal tape, whose contents is always a constructive ordinal. Given a language on its input tape, $M$ runs forever, producing infinitely many outputs as usual, except that just before $M$ changes its mind, if $\alpha$ is currently on its ordinal tape, $M$ is required to compute some ordinal $\beta<\alpha$ and replace the contents of the ordinal tape with $\beta$ before proceeding to change its mind. (So if $\alpha=0$, no mind-change may take place.) $M$ may alter its ordinal tape at any other time, but the only allowed change is replacement with a lesser ordinal.

\footnotetext{
${ }^{4}$ The usual expression for the rank of $a$ is $|a|$, but we change the notation here to avoid confusion with set cardinality and string length.
} 
Thus a procrastinating learner must decrease its ordinal tape before each mindchange.

We abuse notation and let $M_{1}, M_{2}, \ldots$ be a standard enumeration of procrastinating learners. Such an effective enumeration exists because we can enforce the ordinal-decrease requirement for a machine's ordinal tape: if $b \in \mathrm{ORD}$ is the current contents of the ordinal tape, and the machine wishes (or is required) to alter it - say, to some value $a \in \mathbb{N}$-we first start to computably enumerate the set of all $c \in \mathrm{ORD}$ such that $\|c\|<\|b\|$ and allow the machine to proceed only when $a$ shows up in the enumeration.

Definition 5.24. Let $M$ be a procrastinating learner, $\alpha$ a constructive ordinal, and $A$ a language. We say that $M$ learns $\operatorname{SUBSEQ}(A)$ with $\alpha$ mind-changes if $M$ learns $\operatorname{SUBSEQ}(A)$ with $\alpha$ initially on its ordinal tape.

If $\mathcal{C}$ is a class of languages, we say that $\mathcal{C} \in \mathrm{SUBSEQ} \mathrm{EX}_{\alpha}$ if there is a procrastinating learner that learns every language in $\mathcal{C}$ with $\alpha$ mind-changes.

The following two observations are straightforward and given without proof.

OBSERvation 5.25. If $\alpha<\omega$, then SUBSEQ-EX is $_{\alpha}$ the same as the usual finite mind-change version of SUBSEQ-EX.

Observation 5.26. For all $\alpha<\beta<\omega_{1}^{\mathrm{CK}}$,

$$
\text { SUBSEQ-EX }{ }_{\alpha} \subseteq \text { SUBSEQ-EX }{ }_{\beta} \subseteq \text { SUBSEQ-EX. }
$$

In [13], Freivalds and Smith defined $\mathrm{EX}_{\alpha}$ for constructive $\alpha$ and showed that this hierarchy separates using classes of languages constructed by a noneffective diagonalization based on learner behavior. Although their technique can be

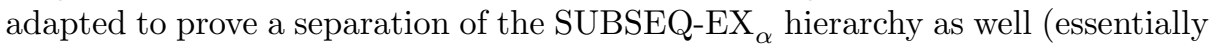
by trading step positions in the step function for strings in the language), we take a different approach and define straightforward, learner-independent classes of languages that separate the SUBSEQ-EX ${ }_{\alpha}$ hierarchy. These or similar classes may be of independent interest.

Definition 5.27. For every $\alpha<\omega_{1}^{\mathrm{CK}}$, we define the class $\mathcal{F}_{\alpha}$ inductively as follows: Let $n=N(\alpha)$, and let $\lambda=\Lambda(\alpha)$.

- If $\lambda=0$, let

$$
\mathcal{F}_{\alpha}=\mathcal{F}_{n}=\left\{B \cup+\emptyset:\left(B \subseteq 0^{*}\right) \wedge(|B| \leq n)\right\} .
$$

- If $\lambda>0$, then $\lambda$ has notation $3 \cdot 5^{e}$ for some TM index $e$. Let

$$
\mathcal{F}_{\alpha}=\left\{B \cup+C:\left(B, C \subseteq 0^{*}\right) \wedge(|B| \leq n+1) \wedge\left(C \in \mathcal{F}_{M_{e}(m(B))}\right)\right\} .
$$

It is evident by induction on $\alpha$ that $\mathcal{F}_{\alpha}$ consists only of finite unary languages and that $\emptyset \in \mathcal{F}_{\alpha}$. Note that in the case of finite $\alpha$ we have the condition $|B| \leq n$, but in the case of $\alpha \geq \omega$ we have the condition $|B| \leq n+1$. This is not a mistake.

The proofs of the next two theorems are roughly analogous to the finite mindchange case, but they are complicated somewhat by the need for effective transfinite recursion.

THEOREM 5.28. For every constructive $\alpha, \mathcal{F}_{\alpha} \in \mathrm{SUBSEQ}_{\mathrm{EX}}$. In fact, there is a single procrastinating learner $Q$ such that for every $\alpha, Q$ learns every language in $\mathcal{F}_{\alpha}$ with $\alpha$ mind-changes. 
Proof. With $\alpha$ initially on its ordinal tape and language $A \in \mathcal{F}_{\alpha}$ on its input tape, for $n=0,1,2, \ldots$, the machine $Q$ checks whether $0^{n} \in A$. If not, then $Q$ simply outputs the index $k$ such that $L\left(F_{k}\right)=\operatorname{SUBSEQ}\left(A \cap 0^{<n+1}\right)$, which is the same as its previous output, if there was one. If $0^{n} \in A$, then outputting $k$ as above may require a mind-change first. There are two cases:

1. If $N(\alpha)>0$, then $Q$ replaces $\alpha$ with its predecessor, outputs $k$ as above, and goes on to the next $n$.

2. If $N(\alpha)=0$, then it must be that $\alpha \geq \omega$ (otherwise, $A \notin \mathcal{F}_{\alpha}$ ), and so $\alpha$ $(=\Lambda(\alpha))$ has notation $3 \cdot 5^{e}$ for some $e$. $Q$ then

(a) computes $B:=\xi\left(A \cap 0^{<n+1}\right)$ and $\gamma:=M_{e}(m(B))$ (note: it must be the case that $B=\xi(A)$; otherwise, $\left.A \notin \mathcal{F}_{\alpha}\right)$, then

(b) changes its ordinal tape to $\gamma+1$, outputs $k$ as above, then changes its ordinal tape again to $\gamma$, then

(c) simulates itself recursively (forever) from the beginning with $C:=$ $\pi(m(B) ; A)$ on the input tape and $\gamma$ initially on the ordinal tape. Whenever the simulation decreases its ordinal tape, $Q$ decreases its own ordinal tape to the same value, and whenever the simulation outputs an index $i, Q$ outputs the index of a DFA for $\operatorname{SUBSEQ}\left(B \cup+\left(L\left(F_{i}\right) \cap 0^{*}\right)\right)$ instead.

A straightforward induction on $\alpha$ proves that $Q$ correctly learns $\operatorname{SUBSEQ}(A)$ with $\alpha$ mind-changes for any $A \in \mathcal{F}_{\alpha}$.

If $\alpha<\omega$, then $A=B \cup$ + $\emptyset$ for some $B \subseteq 0^{*}$ such that $|B| \leq N(\alpha)=\alpha$. Because $|A|=|B| \leq N(\alpha), Q$ has enough mind-changes available to repeat Case 1 until it sees all of $A$ and thus learns $\operatorname{SUBSEQ}(A)$.

Now suppose that $\alpha \geq \omega$ and that $\Lambda(\alpha)$ has notation $3 \cdot 5^{e}$ for some $e$. We know that $A=B \cup+C$, where $B, C \subseteq 0^{*},|B| \leq N(\alpha)+1$, and $C \in \mathcal{F}_{\gamma}$, where $\gamma=M_{e}(m(B))$. Since $|B| \leq N(\alpha)+1, Q$ can repeat Case 1 enough times to see all but the longest string of $B$ (if there is one) without dropping its ordinal below $\Lambda(\alpha)$. Therefore, if or when $Q$ encounters Case 2 and needs to drop its ordinal below $\Lambda(\alpha)$, it has seen all of $B$, which is thus computed correctly along with $\gamma$ in Step 2a. Since $C \in \mathcal{F}_{\gamma}$, by the inductive hypothesis, the recursive simulation in Step 2c correctly learns $\operatorname{SUBSEQ}(C)$ with $\gamma$ mind-changes, and so $Q$ has enough mind-changes available to run the simulation, which eventually converges on an index $i$ such that $L\left(F_{i}\right)=\operatorname{SUBSEQ}(C)=0^{<m(C)}$. Clearly,

$$
\operatorname{SUBSEQ}(A)=\operatorname{SUBSEQ}(B \cup+C)=\operatorname{SUBSEQ}(B \cup+\operatorname{SUBSEQ}(C)) .
$$

So the original run of $Q$ will output the index of a DFA recognizing $\operatorname{SUBSEQ}(A)$ cofinitely often, using $\alpha$ mind-changes.

There is one last technicality. Note that $Q$ decreases its ordinal just before starting the simulation in Step 2c. This is needed because the first conjecture of the simulation may produce a mind-change in the original run of $Q$.

TheOREM 5.29. For all $\beta<\alpha<\omega_{1}^{\mathrm{CK}}, \mathcal{F}_{\alpha} \notin$ SUBSEQ-EX $_{\beta}$. In fact, there is a computable function $r$ such that, for each $e$ and $\beta<\alpha<\omega_{1}^{\mathrm{CK}}, M_{r(e, \alpha, \beta)}$ is total and decides a language $A_{e, \alpha, \beta}=L\left(M_{r(e, \alpha, \beta)}\right) \in \mathcal{F}_{\alpha}$ such that $M_{e}$ does not learn $\operatorname{SUBSEQ}\left(A_{e, \alpha, \beta}\right)$ with $\beta$ mind-changes. 
Proof. This proof generalizes the proof of Theorem 5.22 to the transfinite case. We first define a computable function $v(e, c, t, b)$ such that for all $e, c, t, b \in$ $\mathbb{N}$, the procrastinating learner $M_{v(e, c, t, b)}$ with language $C$ on its input tape and $g \in \mathbb{N}$ on its ordinal tape ${ }^{5}$ behaves as follows:

1. Without changing the ordinal tape or outputting anything, $M_{v(e, c, t, b)}$ simulates $M_{e}$ for $t$ steps with $\left(D_{c} \cap 0^{*}\right) \cup+\left(C \cap 0^{*}\right)$ on $M_{e}$ 's input tape and $b$ on $M_{e}$ 's ordinal tape.

2. $M_{v(e, c, t, b)}$ continues to simulate $M_{e}$ as above beyond $t$ steps, except that now:

- Whenever $M_{e}$ changes its ordinal tape to some value $u, M_{v(e, c, t, b)}$ changes its ordinal tape to the same value $u$ (provided this is allowed).

- Whenever $M_{e}$ outputs a value $k, M_{v(e, c, t, b)}$ outputs the index of a DFA recognizing the language $\pi\left(m\left(D_{c}\right) ; L\left(F_{k}\right)\right)$ (provided this is allowed).

The function $v$ is defined so that if $M_{e}$ learns $\operatorname{SUBSEQ}\left(D_{c} \cup+C\right.$ ) (for some $\left.D_{c}, C \subseteq 0^{*}\right)$ with $\beta$ mind-changes and $M_{e}$ manages to decrease its ordinal tape to some $\delta$ within the first $t$ steps of its computation, then $M_{v(e, c, t, \beta)}$ learns $\operatorname{SUBSEQ}(C)$ with $\gamma$ mind-changes, for any $\gamma \geq \delta$. (Observe that SUBSEQ $(C)=$ $\pi\left(m\left(D_{c}\right)\right.$; SUBSEQ $\left.\left(D_{c} \cup+C\right)\right)$.) We will use the contrapositive of this fact in the proof, below.

Given $e$ and $\beta<\alpha<\omega_{1}^{\mathrm{CK}}$ we construct the set $A_{e, \alpha, \beta} \subseteq 0^{*}$, which is decidable uniformly in $e, \alpha, \beta$. The rough idea is that we build $\bar{A}_{e, \alpha, \beta}$ to be of the form $B \cup+C$, where $B, C \subseteq 0^{*}$ and $|B| \leq N(\alpha)+1$ (assuming $\alpha \geq \omega$ ), while diagonalizing against $M_{e}$ with $\beta$ on its ordinal tape. We put strings into $B$ to force mind-changes in $M_{e}$ until either $M_{e}$ runs out of mind-changes (and is wrong) or it decreases its ordinal tape to some ordinal $\delta<\Lambda(\alpha)$. If the latter happens, we then put one more string into $B$ to code some $\gamma$ such that $\delta<\gamma<\Lambda(\alpha)$, and then (recursively) make $C$ equal to $A_{\hat{e}, \gamma, \delta}$ for some appropriate $\hat{e}$ chosen using the function $v$, above. Here is the construction of $A_{e, \alpha, \beta}$ :

1. Let $\lambda=\Lambda(\alpha)$.

2. Initialize $B:=\emptyset$ and $t:=0$.

3. Repeat the following as necessary to construct $B$ :

(a) Run $M_{e}$ with $B \cup+\emptyset$ on its tape and $\beta$ initially on its ordinal tape until it outputs some $k$ such that $L\left(F_{k}\right)=\operatorname{SUBSEQ}(B \cup+\emptyset)$ after more than $t$ steps. This may never happen, in which case we define $A_{e, \alpha, \beta}:=B \cup+\emptyset$ and we are done.

(b) Let $t^{\prime}>t$ be the number of steps it took $M_{e}$ to output $k$, above. Let $\delta$ be the contents of $M_{e}$ 's ordinal tape when $k$ was output. [Note that $M_{e}$ did not have time to scan any strings of the form $0^{s}$ for $s>t^{\prime}$.] Reset $t:=t^{\prime}$.

(c) If $\delta<\lambda$, then go on to Step 4 .

(d) Set $B:=B \cup\left\{0^{t+1}\right\}$ and continue the repeat-loop.

4. Now we have $\delta<\lambda$, and so $\lambda$ is a limit ordinal with notation $3 \cdot 5^{u}$ for some $u$. Let $p$ be least such that $p>t$ and $M_{u}(p+1)$ is the notation for some ordinal $\gamma>\delta$. [Note that $\gamma<\lambda \leq \alpha$.]

\footnotetext{
${ }^{5}$ For the purposes of defining the function $v$, we must take $b$ and $g$ to be arbitrary numbers, although they will usually be notations for ordinals.
} 
5. Set $B:=B \cup\left\{0^{p}\right\}$. [This makes $m(B)=p+1$.]

6 . Let $c$ be such that $B=D_{c}$. Set $\hat{e}:=v(e, c, t, \beta)$, and (recursively) define $A_{e, \alpha, \beta}:=B \cup+A_{\hat{e}, \gamma, \delta}$. [The ordinal in the second subscript decreases from $\alpha$ to $\gamma$, so the recursion is well-founded.]

For all $e$ and all $\beta<\alpha<\omega_{1}^{\mathrm{CK}}$, we show by induction on $\alpha$ that $A_{e, \alpha, \beta} \in \mathcal{F}_{\alpha}$ and that $M_{e}$ cannot learn $\operatorname{SUBSEQ}\left(A_{e, \alpha, \beta}\right)$ with $\beta$ initially on its ordinal tape. Let $\lambda=\Lambda(\alpha)$ ( $\lambda$ may be either 0 or a limit), and let $n=N(\alpha)$. Consider $M_{e}$ running with $A_{e, \alpha, \beta}$ on its input tape and $\beta$ initially on its ordinal tape. In the repeat-loop, $t$ bounds the running time of $M_{e}$ and strictly increases from one complete iteration to the next, and the only strings added to $B$ have length greater than $t$. This implies two things: (1) that $M_{e}$ behaves the same in Step 3a with $B \cup+\emptyset$ on its tape as it would with $A_{e, \alpha, \beta}$ on its tape, and (2) the number of mind-changes $M_{e}$ must make to be correct increases in each successive iteration of the loop.

We now consider two cases:

$\boldsymbol{\lambda}$ is the $\mathbf{0}$ ordinal: Then $M_{e}$ can change its mind at most $n-1$ times (since $\beta<\alpha=n)$. This means that the repeat-loop will run for at most $n$ complete iterations, then hang in Step 3a on the next iteration, because by then $M_{e}$ has run out of mind-changes and so cannot update its answer to be correct. In this case, $A_{e, \alpha, \beta}=B \cup+\emptyset$, and we've added at most $n$ strings to $B$. Thus $A_{e, \alpha, \beta} \in \mathcal{F}_{\alpha}$, and $M_{e}$ does not learn $\operatorname{SUBSEQ}\left(A_{e, \alpha, \beta}\right)$ with $\beta$ mind-changes.

$\boldsymbol{\lambda}$ is a limit ordinal with notation $3 \cdot \mathbf{5}^{\boldsymbol{u}}$ for some $\boldsymbol{u}$ : In this case, $M_{e}$ can change its mind at most $n-1$ times before it must drop its ordinal to some $\delta<\lambda$ for its next mind-change. So again there can be at most $n$ complete iterations of the repeat-loop - putting at most $n$ strings into $B$-before we either hang in Step 3a (which is just fine) or go on to Step 4. In the latter case, we put one more string into $B$ in Step 5, making $|B| \leq n+1$. By the inductive hypothesis and the choice of $p$ and $\gamma$, we have $A_{\hat{e}, \gamma, \delta} \in \mathcal{F}_{\gamma}=\mathcal{F}_{M_{u}(m(B))}$, and so $A_{e, \alpha, \beta} \in \mathcal{F}_{\alpha}$.

The index $\hat{e}$ is chosen precisely so that if $M_{e}$ learns $\operatorname{SUBSEQ}\left(A_{e, \alpha, \beta}\right)$ with $\beta$ mind-changes then $M_{\hat{e}}$ learns $\operatorname{SUBSEQ}\left(A_{\hat{e}, \gamma, \delta}\right)$ with $\delta$ mind-changes. By the inductive hypothesis, $M_{\hat{e}}$ cannot do this. Thus in either case $M_{e}$ does not learn $\operatorname{SUBSEQ}\left(A_{e, \alpha, \beta}\right)$ with $\beta$ mind-changes.

It remains to show that $A_{e, \alpha, \beta}$ is decidable uniformly in $e, \alpha, \beta$. The only tricky part is Step 3a, which may run forever. It is not hard to see, however, that if $M_{e}$ runs for at least $\ell$ steps for some $\ell$, then either $0^{\ell}$ is already in $B$ by this point or it will never get into $B$. Hence we can decide whether or not $0^{2 \ell+1}$ is in $A_{e, \alpha, \beta}$. Even-length strings in $0^{*}$ can be handled similarly, possibly via a recursive call to $A_{\hat{e}, \gamma, \delta}$.

We end with an easy observation.

Corollary 5.30.

SUBSEQ-EX $\nsubseteq \bigcup_{\alpha<\omega_{1}^{\mathrm{CK}}}$ SUBSEQ-EX ${ }_{\alpha}$ 
Proof. Let $\mathcal{F} \in$ SUBSEQ-EX be the class of Notation 4.16. For all $\alpha<\omega_{1}^{\mathrm{CK}}$, we clearly have $\mathcal{F}_{\alpha+1} \subseteq \mathcal{F}$, and so $\mathcal{F} \notin$ SUBSEQ-EX by $_{\alpha}$ Theorem 5.29.

5.5. Teams. In this section, we show that $[a, b]$ SUBSEQ-EX depends only on $\lfloor b / a\rfloor$. The next lemma is analogous to a result of Pitt \& Smith concerning teams in BC and EX learning [29, Theorem 12], proved using techniques of Pitt [28] regarding probabilistic inference. Because testing language equivalence of DFAs is trivial, we can avoid most of the complexity in their proof and give a new, easy proof of Lemma 5.31.

LEMMA 5.31. For all $1 \leq a \leq b$,

$$
[a, b] \mathrm{SUBSEQ}-\mathrm{EX}=[1,\lfloor b / a\rfloor] \mathrm{SUBSEQ}-\mathrm{EX} .
$$

Proof. Let $q=\lfloor b / a\rfloor$. We get $[1, q]$ SUBSEQ-EX $\subseteq[a, b]$ SUBSEQ-EX via the standard trick of duplicating each of the $q$ learners in a team $a$ times, then observing that $[a, q a]$ SUBSEQ-EX $\subseteq[a, b]$ SUBSEQ-EX.

For the reverse containment, let $Q_{1}, \ldots, Q_{b}$ be learners and fix a language $A$ such that at least $a$ of the $Q_{i}$ 's learn $\operatorname{SUBSEQ}(A)$. For any $t>0$, let $k_{1}(t), \ldots, k_{b}(t)$ be the most recent outputs of $Q_{1}, \ldots, Q_{b}$, respectively, after running for $t$ steps with $A$ on their tapes (if some machine $Q_{i}$ has not yet output anything in $t$ steps, let $k_{i}(t)=0$ ).

We define learners $N_{1}, \ldots, N_{q}$ to behave as follows with $A$ on their tapes.

Define a consensus value at time $t$ to be a value that shows up at least $a$ times in the list $k_{1}(t), \ldots, k_{b}(t)$. (Each $N_{j}$ uses $A$ only for computing $k_{1}(t), \ldots, k_{b}(t)$ and nothing else.) We let

$$
\operatorname{POPULAR}(t):=\left\{v:\left|\left\{j \in\{1, \ldots, b\}: k_{j}(t)=v\right\}\right| \geq a\right\}
$$

to be the set of these consensus values. There can be at most $q$ many different consensus values at any given time, so we can make the machines $N_{j}$ output these consensus values. If $k_{\text {correct }}$ is the index of the DFA recognizing $\operatorname{SUBSEQ}(A)$, then $k_{\text {correct }}$ will be a consensus value at all sufficiently large times, and so $k_{\text {correct }}$ will eventually always be output by one or another of the $N_{j}$. The only trick is to ensure that $k_{\text {correct }}$ is eventually output by the same $N_{j}$ each time. To make sure of this, the $N_{j}$ will output consensus values in order of seniority.

For $1 \leq j \leq q$ and $t=1,2,3, \ldots$, each machine $N_{j}$ computes $\operatorname{POPULAR}\left(t^{\prime}\right)$ for all $t^{\prime} \leq t$. For each $v \in \mathbb{N}$, we define the start time of $v$ at time $t$ to be

$$
\operatorname{Start}_{t}(v):= \begin{cases}(\mu s \leq t)\left[v \in \bigcap_{s \leq t^{\prime} \leq t} \operatorname{POPULAR}\left(t^{\prime}\right)\right] & \text { if } v \in \operatorname{POPUlAR}(t), \\ t+1 & \text { otherwise. }\end{cases}
$$

As its $t$ 'th output, $N_{j}$ outputs the value with the $j$ 'th smallest value of $\operatorname{Start}_{t}(v)$. If there is a tie, then we consider the smaller value to have started earlier. This ends the description of the machines $N_{1}, \ldots, N_{q}$.

Let $Y=\bigcup_{s} \bigcap_{t \geq s} \operatorname{POPULAR}(t)$, the set of all consensus values that occur cofinitely often. Clearly, $k_{\text {correct }} \in Y$, and there is a time $t_{0}$ such that all elements of $Y$ are consensus values at all times $t \geq t_{0}$. Note that the start times of the values in $Y$ do not change from $t_{0}$ onward, but the start time of any value not in $Y$ increases monotonically without bound. Thus there is a time $t_{1} \geq t_{0}$ beyond which any $v \notin Y$ has a start time later than that of any $v^{\prime} \in Y$. It follows that from time $t_{1}$ onward, the start time of $k_{\text {correct }}$ has a fixed rank amongst the start 
times of all the current consensus values, and so $k_{\text {correct }}$ is output by the same machine $N_{j}$ at all times $t \geq t_{1}$.

To prove a separation, we cannot use unary languages as we have before; it is easy to see (exercise for the reader) that $\mathcal{P}\left(0^{*}\right) \in[1,2]$ SUBSEQ-EX. To separate the team hierarchy beyond level 2 , we use an alphabet $\Sigma$ that contains 0 and 1 (at least) and show that $\mathcal{S}_{\leq n} \in[1, n+1]$ SUBSEQ-EX $-[1, n]$ SUBSEQ-EX for all $n \geq 1$, where $\mathcal{S}_{\leq n}$ is given in Definition 5.1.

LEMmA 5.32. For all $n \geq 1, \mathcal{S}_{\leq n} \in[1, n+1]$ SUBSEQ-EX and $\mathcal{S}_{\leq n} \cap$ DEC $\notin$ $[1, n]$ SUBSEQ-EX. In fact, there is a computable function $d(s)$ such that for all $n \geq 1$ and all $e_{1}, \ldots, e_{n}$, the machine $M_{d\left(\left[e_{1}, \ldots, e_{n}\right]\right)}$ decides a set $A_{\left[e_{1}, \ldots, e_{n}\right]} \in \mathcal{S}_{\leq n}$ that is not learned by any of $M_{e_{1}}, \ldots, M_{e_{n}} \cdot 6$

Proof. Fix $n \geq 1$. First, we have $\mathcal{S}_{\leq n}=\mathcal{S}_{0} \cup \cdots \cup \mathcal{S}_{n}$, and $\mathcal{S}_{i} \in$ SUBSEQ-EX for each $i \leq n$ by Proposition 5.2. It follows that $\mathcal{S}_{\leq n} \in[1, n+1]$ SUBSEQ-EX.

Next, we show that $\mathcal{S}_{\leq n} \notin[1, n]$ SUBSEQ-EX. Fix any $n$ learners $Q_{1}, \ldots, Q_{n}$. We build a set $A \subseteq \Sigma^{*}$ in stages $n, n+1, n+2, \ldots$, ensuring that $|\operatorname{os}(A)| \leq n$ (hence $A \in \mathcal{S}_{\leq n}$ ) and that none of the $Q_{i}$ learn $\operatorname{SUBSEQ}(A)$. At each stage $j \geq$ $n$, we define $n$ strings $y_{1}^{j}, \ldots, y_{n}^{j} \in\{0,1\}^{*}$ which are candidates for membership in $\operatorname{os}(A)$. These strings satisfy

1. $\left|y_{1}^{j}\right| \leq \cdots \leq\left|y_{n}^{j}\right| \leq j+1$, and

2. $y_{i}^{j} \in 0^{n-i} 1^{*} 1$ for all $1 \leq i \leq n$.

Note that these two conditions imply that $y_{1}^{j}, \ldots, y_{n}^{j}$ are pairwise $\preceq$-incomparable. We then define $A$ on all strings of length $j$.

Stage $n$ : For all $1 \leq i \leq n$, set $y_{i}^{n}:=0^{n-i} 1^{i+1}$. Set $A_{n}:=\Sigma \leq n$.

Stage $j>n$ :

- Run each learner $Q_{i}$ for $j-1$ steps with $A_{j-1}$ on its tape (by the familiar argument, $Q_{i}$ will act the same as with $A$ on its tape), and let $k_{i}$ be its most recent output (or let $k_{i}=0$ if there is no output yet).

- Compute $s_{i}:=\left|\operatorname{os}\left(L\left(F_{k_{i}}\right)\right)\right|$ for all $1 \leq i \leq n$.

- Let $m_{j}$ be the least element of $\{0, \ldots, n\}-\left\{s_{1}, \ldots, s_{n}\right\}$.

- Set $y_{i}^{j}:=y_{i}^{j-1}$ for all $1 \leq i \leq m_{j}$, and set $y_{i}^{j}:=0^{n-i} 1^{j+1-n+i}$ for all $m_{j}<i \leq n$.

- Set $A_{j}:=A_{j-1} \cup\left\{x \in \Sigma^{=j}:\left(\forall i \leq m_{j}\right) y_{i}^{j} \npreceq x\right\}$.

Define $A:=\bigcup_{j=n}^{\infty} A_{j}$. Also define $m:=\liminf _{j \rightarrow \infty} m_{j}$, and let $j_{0}>n$ be least such that $m_{j} \geq m$ for all $j \geq j_{0}$. For $1 \leq i \leq m$, we then have $y_{i}^{j_{0}}=$ $y_{i}^{j_{0}+1}=y_{i}^{j_{0}+2}=\cdots$, and we define $y_{i}$ to be this string. It remains to show that $\operatorname{os}(A)=\left\{y_{1} \ldots, y_{m}\right\}$, for if this is the case, then the obstruction set size $m=|\operatorname{os}(A)|=|\operatorname{os}(\operatorname{SUBSEQ}(A))|$ is omitted infinitely often by all the learners running with $A$ on their tapes, and so none of the learners can converge on a language with an obstruction set of size $m$, and hence none of the learners learn $\operatorname{SUBSEQ}(A)$.

To see that $\operatorname{os}(A)=\left\{y_{1}, \ldots, y_{m}\right\}$, consider an arbitrary string $x \in \Sigma^{*}$. We need to show that $x \in \operatorname{SUBSEQ}(A)$ iff $(\forall i) y_{i} \npreceq x$. By the construction, no $z \succeq y_{i}$ ever enters $A$ for any $i \leq m$, so if $x \preceq z$ and $z \in A$, then $(\forall i) y_{i} \npreceq z$ and thus

\footnotetext{
${ }^{6}\left[e_{1}, e_{2}, \ldots, e_{n}\right]$ is a natural number encoding the finite sequence $e_{1}, e_{2}, \ldots, e_{n}$.
} 
$(\forall i) y_{i} \npreceq x$. Conversely, if $(\forall i) y_{i} \npreceq x$, then $(\forall i) y_{i} \npreceq x 0^{t}$ for any $t \geq 0$, because each $y_{i}$ ends with a 1 . Fix the least $j_{1} \geq \max \left(j_{0},|x|\right)$ such that $m_{j_{1}}=m$, and let $t=j_{1}-|x|$. Then $\left|x 0^{t}\right|=j_{1}$, and $x 0^{t}$ is added to $A$ at Stage $j_{1}$. So we have $x \preceq x 0^{t} \in A$, whence $x \in \operatorname{SUBSEQ}(A)$.

Finally, the whole construction of $A$ above is effective uniformly in $n$ and indices for $Q_{1}, \ldots, Q_{n}$, and uniformly decides $A$. Thus the computable function $d$ of the Lemma exists.

Remark. The foregoing proof can be easily generalized to show that $\mathcal{S}_{j_{1}} \cup \mathcal{S}_{j_{2}} \cup$ $\cdots \cup \mathcal{S}_{j_{k}} \in[1, k]$ SUBSEQ-EX - [1, $\left.k-1\right]$ SUBSEQ-EX for all $j_{1}<j_{2}<\cdots<j_{k}$.

Lemmas 5.31 and 5.32 combine to show the following general theorem, which completely characterizes the containment relationships between the various team learning classes $[a, b]$ SUBSEQ-EX.

THEOREM 5.33. For every $1 \leq a \leq b$ and $1 \leq c \leq d,[a, b]$ SUBSEQ-EX $\subseteq$ $[c, d]$ SUBSEQ-EX if and only if $\lfloor b / a\rfloor \leq\lfloor d / c\rfloor$.

Proof. Let $p=\lfloor b / a\rfloor$ and let $q=\lfloor d / c\rfloor$.

By Lemma 5.31 we have

$$
[a, b] \mathrm{SUBSEQ}-\mathrm{EX}=[1, p] \mathrm{SUBSEQ}-\mathrm{EX},
$$

and

$$
[c, d] \mathrm{SUBSEQ}-\mathrm{EX}=[1, q] \mathrm{SUBSEQ}-\mathrm{EX} .
$$

By Lemma 5.32 we have $[1, p]$ SUBSEQ-EX $\subseteq[1, q]$ SUBSEQ-EX if and only if $p \leq q$.

5.6. Anomalies and teams. In this and the next few subsections we will discuss the effect that combining the variants discussed previously have on the results of the previous subsections.

The next result shows that Theorem 5.17 is unaffected by teams. In fact, teams and anomalies are completely orthogonal.

THEOREM 5.34. The anomaly hierarchy collapses with teams. In other words, for all $a$ and $b$,

$$
[a, b] \mathrm{SUBSEQ} \mathrm{EX}^{*}=[a, b] \mathrm{SUBSEQ}-\mathrm{EX} .
$$

Proof. Given a team $M_{e_{1}}, \ldots, M_{e_{b}}$ of $b$ Turing machines, we use the collapse strategy from Theorem 5.17 on each of the machines. We replace each $M_{e_{i}}$ with the machine $M_{h\left(e_{i}\right)}$, where $h$ is the function of Theorem 5.17. If $a$ of the $b$ machines learn the subsequence language with finite anomalies each, then their replacements will learn it with no anomalies.

5.7. Anomalies and mind-changes. Next, we consider machines which are allowed a finite number of anomalies, but have a bounded number of mindchanges.

In our proof that the anomaly hierarchy collapses (Theorem 5.17), the simulating learner $M_{h(e)}$ may have to make many more mind-changes than the learner $M_{e}$ being simulated. As the next result shows, we cannot do better than this.

Proposition 5.35. SUBSEQ-EX $\nsubseteq_{0}^{*} \bigcup_{c \in \mathbb{N}}$ SUBSEQ-EX $_{c}$. (It is even the case that SUBSEQ-EX $\mathbb{E}_{0}^{*} \nsubseteq \bigcup_{\alpha<\omega_{1}^{\mathrm{CK}}}$ SUBSEQ-EX ${ }_{\alpha}$.) 
Proof. The class $\mathcal{F}$ of Notation 4.16 is in SUBSEQ-EX ${ }_{0}^{*}$ (the learner always outputs the DFA for $\emptyset$ ). But $\mathcal{F} \notin$ SUBSEQ-EX for $_{c}$ any $c \in \mathbb{N}$ by Theorem 5.22 (and $\mathcal{F} \notin$ SUBSEQ-EX $_{\alpha}$ for any $\alpha<\omega_{1}^{\text {CK }}$ by Corollary 5.30).

In light of Proposition 5.35, it may come as a surprise that a bounded number of anomalies may be removed without any additional mind-changes.

TheOREM 5.36. SUBSEQ-EX ${ }_{c}^{a}=$ SUBSEQ-EX $_{c}$ for all $a, c \geq 0$. In fact, there is a computable $h$ such that, for all e, a and languages $A, M_{h(e, a)}$ on $A$ makes no more mind-changes than $M_{e}$ on $A$, and if $M_{e}$ learns $\operatorname{SUBSEQ}(A)$ with at most a anomalies, then $M_{h(e, a)}$ learns $\operatorname{SUBSEQ}(A)$ (with zero anomalies).

Proof. The $\supseteq$-containment is obvious. For the $\subseteq$-containment, we modify the learner in the proof of Theorem 5.17. Given $e$ and $a$, we give the algorithm for the learner $M_{h(e, a)}$ below. We will use the word "default" as a verb to mean, "output the same DFA as we did last time, or, if there was no last time, don't output anything." The opposite of defaulting is "acting." Here's how $M_{h(e, a)}$ works:

When language $A$ is on the tape:

1. Run $M_{e}$ with $A$. Wait for $M_{e}$ to output something.

2. Whenever $M_{e}$ outputs some hypothesis $k$, do the following:

(a) Let $n$ be the number of times $M_{e}$ has output a hypothesis thus far. ( $k$ is the $n$th hypothesis.)

(b) If there was some time in the past when we acted and $M_{e}$ has not changed its mind since then, then default.

(c) Else, if $F_{k}$ has more than $n$ states, then default.

(d) Else, if $L\left(F_{k}\right) \cup \Sigma^{<n}$ is not $\preceq$-closed, then default.

(e) Else, if there are strings $w \in \operatorname{os}\left(L\left(F_{k}\right) \cup \Sigma^{<n}\right)$ and $z \in A$ such that $w \preceq z$ and $|z|<|w|+a$, then default. [Note that $w$, if it exists, has length at least $n$.]

(f) Else, find a DFA $G$ recognizing the language

$$
\operatorname{SUBSEQ}\left(\left(A \cap \Sigma^{<n}\right) \cup\left(L\left(F_{k}\right) \cap \Sigma^{\geq n}\right)\right) \text {, }
$$

and output the index of $G$. [This is where we act, i.e., not default.]

First, it is not too hard to see that $M_{h(e, a)}$ does not change its mind any more than $M_{e}$ does: After $M_{e}$ makes a new conjecture, $M_{h(e, a)}$ will act at most once before $M_{e}$ makes a different conjecture. This is ensured by Step 2b. Note that $M_{h(e, a)}$ only makes a new conjecture when it acts.

Suppose $M_{e}$ learns SUBSEQ $(A)$ with at most $a$ anomalies. Let $F$ be the final DFA output by $M_{e}$ with $A$ on its tape. We have $|L(F) \triangle \operatorname{SUBSEQ}(A)| \leq a$. Let $n_{0}$ be least such that $M_{e}$ always outputs $F$ starting with its $n_{0}$ th hypothesis onwards. It remains to show that

1. $M_{h(e, a)}$ acts sometime after $M_{e}$ starts perpetually outputting $F$, i.e., after its $n_{0}$ th hypothesis, and

2. when this happens, the $G$ output by $M_{h(e, a)}$ is correct, that is, $L(G)=$ $\operatorname{SUBSEQ}(A)$. (Since $M_{h(e, a)}$ only defaults thereafter, it outputs $G$ forever and thus learns $\operatorname{SUBSEQ}(A)$.)

For (1), we start by noting that there is a least $n \geq n_{0}$ such that 
- $F$ has at most $n$ states, and

- all anomalies are of length less than $n$, i.e., $L(F) \triangle \operatorname{SUBSEQ}(A) \subseteq \Sigma^{<n}$.

We claim that $M_{h(e, a)}$ acts sometime between $M_{e}$ 's $n_{0}$ th and $n$th hypotheses, inclusive. Suppose we've reached $M_{e}$ 's $n$th hypothesis and we haven't acted since the $n_{0}$ th hypothesis. Then we don't default in Step $2 \mathrm{~b}$. We don't default in Step 2c because $F$ has at most $n$ states. Since all anomalies are in $\Sigma^{<n}$, clearly, $L(F) \cup \Sigma^{<n}=\operatorname{SUBSEQ}(A) \cup \Sigma^{<n}$, which is $\preceq$-closed, so we don't default in Step 2d. Finally, we won't default in Step 2e: if $w$ and $z$ existed, then $w$ would be an anomaly of length $\geq n$, but all anomalies are of length $<n$. Thus we act on $M_{e}$ 's $n$th hypothesis, which proves (1).

For (2), we know from (1) that $M_{h(e, a)}$ acts on $M_{e}$ 's $n$th hypothesis, for some $n \geq n_{0}$, at which time $M_{h(e, a)}$ outputs some DFA $G$. We claim that $L(G)=$ $\operatorname{SUBSEQ}(A)$.

Since $M_{h(e, a)}$ acts on $M_{e}$ 's $n$th hypothesis, we know that

- $F$ has at most $n$ states,

- $L(F) \cup \Sigma^{<n}$ is $\preceq$-closed, and

- there are no strings $w \in \operatorname{os}\left(L(F) \cup \Sigma^{<n}\right)$ and $z \in \Sigma^{<|w|+a} \cap A$ such that $w \preceq z$.

It suffices to show that there are no anomalies of length $\geq n$, for then we have

$$
\begin{aligned}
& L(G)=\operatorname{SUBSEQ}\left(\left(A \cap \Sigma^{<n}\right) \cup\left(L(F) \cap \Sigma^{\geq n}\right)\right) \\
& =\operatorname{SUBSEQ}\left(\left(A \cap \Sigma^{<n}\right) \cup\left(\operatorname{SUBSEQ}(A) \cap \Sigma^{\geq n}\right)\right)=\operatorname{SUBSEQ}(A)
\end{aligned}
$$

as in the proof of Theorem 5.17.

There are two kinds of anomalies - false positives (which are elements of $L(F)$ $\operatorname{SUBSEQ}(A)$ ) and false negatives (which are elements of $\operatorname{SUBSEQ}(A)-L(F)$ ).

First, there can be no false positives of length $\geq n$ : Suppose $w$ is such a string. Then since $w$ is at least as long as the number of states of $F$, by the Pumping Lemma for regular languages there are strings $x, y, z$ with $|y|>0$ such that the strings

$$
w=x y z \prec x y^{2} z \prec x y^{3} z \prec \cdots
$$

are all in $L(F)$. But since $w \notin \operatorname{SUBSEQ}(A)$, none of these other strings is in $\operatorname{SUBSEQ}(A)$ either. This means there are infinitely many anomalies, which is false by assumption. Thus no such $w$ exists.

Finally, we prove that there are no false negatives in $\Sigma \geq n$. Suppose $u$ is such a string. We have $u \in \operatorname{SUBSEQ}(A)$, and so there is a string $z \in A$ such that $u \preceq z$. We also have $u \notin L(F) \cup \Sigma^{<n}$, and since $L(F) \cup \Sigma^{<n}$ is $\preceq$-closed, there is some string $w \in \operatorname{os}\left(L(F) \cup \Sigma^{<n}\right)$ such that $w \preceq u$. Now $w \preceq z$ as well, so it must be that $|z| \geq|w|+a$ by what we know above. Since $w \preceq z$, there is also an ascending chain of strings

$$
w=w_{0} \prec w_{1} \prec \cdots \prec w_{k}=z,
$$

where $\left|w_{i}\right|=|w|+i$ and so $k \geq a$. All the $w_{i}$ are in $\operatorname{SUBSEQ}(A)$ because $z \in A$. Moreover, none of the $w_{i}$ are in $L(F) \cup \Sigma^{<n}$ because $w \notin L(F) \cup \Sigma^{<n}$ and $L(F) \cup \Sigma^{<n}$ is $\preceq$-closed. Thus the $w_{i}$ are all anomalies, and there are at least $a+1$ of them, contradicting the fact that $M_{e}$ learns $\operatorname{SUBSEQ}(A)$ with $\leq a$ anomalies. Thus no such $u$ exists. 
Proposition 5.21 and Theorems 5.22 and 5.36 together imply that we cannot replace a single mind-change by any fixed finite number of anomalies. A stronger statement is true.

Theorem 5.37. SUBSEQ-EX S $_{c}$ SUBSEQ-EX $_{c-1}^{*}$ for any $c>0$.

Proof. Let $R_{i}=\left(0^{*} 1^{*}\right)^{i}$ as in Definition 4.8, and define

$$
\mathcal{R}_{c}=\left\{A \subseteq\{0,1\}^{*}: \begin{array}{ll}
A \subseteq R_{c} \wedge \\
& (\exists j)\left[0 \leq j \leq c \wedge \text { is }\left\{R_{j} \subseteq A \subseteq^{*} R_{j}\right]\right.
\end{array}\right\} .
$$

Recall (Notation 4.7) that $A \subseteq{ }^{*} B$ means that $A-B$ is finite.

We claim that $\mathcal{R}_{c} \in$ SUBSEQ-EX ${ }_{c}-$ SUBSEQ-EX $_{c-1}^{*}$ for all $c>0$.

To see that $\mathcal{R}_{c} \in \mathrm{SUBSEQ}-\mathrm{EX}_{c}$, with $A \in \mathcal{R}_{c}$ on the tape the learner $M$ first sets $i:=c$ and may decrement $i$ as the learning proceeds. For each $i$, the machine $M$ proceeds on the assumption that $R_{i} \subseteq A$. For $n=1,2,3, \ldots, M$ waits until $n \geq 2 i$ and there are no strings in $A-R_{i}$ of length $n$. At this point, it is not hard to see that $A-R_{i} \subseteq \Sigma^{<n}$. (Note that a string $x \in\{0,1\}^{*}$ is in $R_{i}$ iff $0 x 1$ has at most $i$ occurrences of 01 as a substring.) $M$ now starts outputting a DFA for $R_{i} \cup\left(A \cap \Sigma^{<n}\right)=\operatorname{SUBSEQ}\left(R_{i} \cup\left(A \cap \Sigma^{<n}\right)\right)$. If $M$ ever discovers a string in $R_{i}-A$, then $M$ resets $i:=i-1$ and starts over. Thus $M$ can make at most $c$ mind-changes before finding the unique $j$ such that $R_{j} \subseteq A \subseteq{ }^{*} R_{j}$.

To show that $\mathcal{R}_{c} \notin$ SUBSEQ-EX ${ }_{c-1}^{*}$ we use a (by now) standard diagonalization. Given a learner $M$, we build $A$ such that $A \cap \Sigma^{<n}=R_{c} \cap \Sigma^{<n}$ for increasing $n$ until $M$ outputs some DFA $F$ such that $L(F)={ }^{*} R_{c}$ while only querying strings of length less than $n$. We then make $A$ look like $R_{c-1}$ on strings of length $\geq n$ until $M$ outputs a DFA $G$ with $L(G)={ }^{*} R_{c-1}$. We then make $A$ look like $R_{c-2}$ above the queries made by $M$ so far, et cetera. In the end, $M$ clearly must make at least $c$ mind-changes to be right within a finite number of anomalies. We can make $A$ decidable uniformly in $c$ and an index for $M$.

Although we don't get any trade-offs between anomalies and mind-changes, we $d o$ get trade-offs between anomaly revisions and mind-changes. If a learner is allowed to revise its bound on allowed anomalies from time to time, then we can trade these revisions for mind-changes. The proper setting for considering anomaly revisions is that of transfinite anomalies, which we consider next.

5.7.1. Transfinite anomalies and mind-changes. This section uses some of the concepts introduced in the section on transfinite mind-changes, above. If you skipped that section, then you may skip this one, too.

We get a trade-off between anomalies and mind-changes if we consider the notion of transfinite anomalies, which we now describe informally. Suppose we have a learner $M$ with a language $A$ on its tape and some constructive ordinal $\alpha<\omega_{1}^{\text {CK }}$ initially on its ordinal tape, and suppose that $M$ can decrease its ordinal any time it wants to (it is not forced to by mind-changes). We say that $M$ learns $\operatorname{SUBSEQ}(A)$ with $\alpha$ anomalies if $M$ 's final DFA $F$ and final ordinal $\beta$ are such that $|L(F) \triangle \operatorname{SUBSEQ}(A)| \leq N(\beta)$. For example, if $M$ starts out with $\omega+\omega$ on its ordinal tape, then at some point after examining $A$ and making conjectures, $M$ may tentatively decide that it can find $\operatorname{SUBSEQ}(A)$ with at most 17 anomalies. It then decreases its ordinal to $\omega+17(N(\omega+17)=17)$. Later, 
$M$ may find that it really needs 500 anomalies. It can then decrease its ordinal a second time from $\omega+17$ to 500 . $M$ is now committed to at most 500 anomalies, because it cannot further increase its allowed anomalies by decreasing its ordinal.

More generally, if $M$ starts with the ordinal $\omega \cdot n+k$ for some $n, k \in \mathbb{N}$, then $M$ is allowed $k$ anomalies to start, and $M$ can increase the number of allowed anomalies up to $n$ many times.

There was no reason to introduce transfinite anomalies before, because the anomaly hierarchy collapses completely. Transfinite anomalies are nontrivial, however, when combined with limited mind-changes.

The next theorem generalizes Theorem 5.36 to the transfinite. It shows that a finite number of extra anomalies makes no difference.

THEOREM 5.38. Let $k, c \in \mathbb{N}$ and let $\lambda<\omega_{1}^{\mathrm{CK}}$ be any limit ordinal. Then SUBSEQ-EX ${ }_{c}^{\lambda+k}=$ SUBSEQ-EX ${ }_{c}^{\lambda}$.

Proof Sketch. We show the $c=0$ case; the general case is similar. Suppose $M$ learns $\operatorname{SUBSEQ}(A)$ with $\lambda+k$ anomalies and no mind-changes. To learn $\operatorname{SUBSEQ}(A)$ with $\lambda$ anomalies and no mind-changes, we first run the algorithm of Theorem 5.36 with $\lambda$ initially on our ordinal tape and assuming $\leq k$ anomalies (i.e., setting $M_{e}:=M$ and $a:=k$ ). If $M$ never drops its ordinal below $\lambda$, then this works fine. Otherwise, at some point, $M$ drops its ordinal to some $\gamma<\lambda$. If this happens before we act-i.e., before we output anything - then we abandon the algorithm, drop our own ordinal to $\gamma$, and from now on simulate $M$ directly. If the drop happens after we act, then $M$ has already outputted some final DFA $F$ and we have outputted some $G$ recognizing $L(G)=\operatorname{SUBSEQ}\left(\left(A \cap \Sigma^{n}\right) \cup\left(L(F) \cap \Sigma^{\geq n}\right)\right)$ for some $n$. Since $L(F) \cup \Sigma^{<n}$ is $\preceq$-closed, it follows that $L(G) \triangle L(F) \subseteq \Sigma^{<n}$ and hence is finite. So we compute $\bar{d}:=|L(G) \triangle L(F)|$, drop our ordinal from $\lambda$ to $\gamma+d$, and keep outputting $G$ forever. Whenever $M$ drops its ordinal further to some $\delta$, then we drop ours to $\delta+d$, etc. If $\ell$ is the final number of anomalies allowed by $M$, then we have

$|L(G) \triangle \operatorname{SUBSEQ}(A)| \leq|L(G) \triangle L(F)|+|L(F) \triangle \operatorname{SUBSEQ}(A)| \leq d+\ell$, and so we have given ourselves enough anomalies.

We show next that $\omega$ anomalies can be traded for an extra mind-change.

TheOrem 5.39. For all $c \in \mathbb{N}$ and $\lambda<\omega_{1}^{\mathrm{CK}}$, if $\lambda$ is zero or a limit, then

$$
\text { SUBSEQ-EX }{ }_{c}^{\lambda+\omega} \subseteq \text { SUBSEQ-EX }{ }_{c+1}^{\lambda} \text {. }
$$

Proof Sketch. Suppose $M$ learns $\operatorname{SUBSEQ}(A)$ with $\lambda+\omega$ anomalies and $c$ mind-changes. With ordinal $\lambda$ on our ordinal tape, we start out by simulating $M$ exactly - outputting the same conjectures - until $M$ drops its ordinal to some $\gamma$. If $\gamma<\lambda$, then we drop our ordinal to $\gamma$ and keep simulating $M$ forever. If $\gamma=\lambda+k$ for some $k \in \mathbb{N}$, then we immediately adopt the strategy in the proof of Theorem 5.38, above. Our first action after this point may constitute an extra mind-change, but that's okay because we have $c+1$ mind-changes available. $\dashv$

Corollary 5.40. SUBSEQ-EX $c_{c}^{\omega \cdot n+k} \subseteq$ SUBSEQ-EX $_{c+n}^{0}$ for all $c, n, k \in \mathbb{N}$.

Proof. By Theorems 5.36, 5.38, and 5.39.

Next we show that the trade-off in Corollary 5.40 is tight. 
TheOREM 5.41. SUBSEQ-EX ${ }_{c}^{\omega \cdot n} \nsubseteq$ SUBSEQ-EX $_{c+n-1}$ for any $c$ and $n>0$.

Proof sketch. Consider the classes $\mathcal{C}_{i}=\left\{A \subseteq 0^{*}:|A| \leq i\right\}$ of Definition 5.20. By Theorem 5.22, $\mathcal{C}_{c+n} \notin$ SUBSEQ-EX $_{c+n-1}$. We check that $\mathcal{C}_{c+n} \in \mathrm{SUBSEQ} \mathrm{EX}_{c}^{\omega \cdot n}$. Given $A \in \mathcal{C}_{c+n}$ on the tape and $\omega \cdot n$ initially on its ordinal tape, the learner $M$ outputs a DFA for $\operatorname{SUBSEQ}\left(A \cap \Sigma^{\leq i}\right)$ as its $i$ th output (as in Proposition 4.17) until it runs out of mind-changes. $M$ continues outputting the same DFA, but every time it finds a new element $0^{j} \in A$ it revises its anomaly count to $j+1$. It can do this $n$ times.

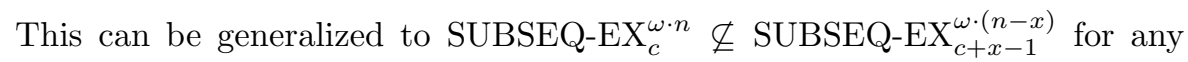
$n \in \mathbb{N}$ and $0 \leq x \leq n$, witnessed by the same class $\mathcal{C}_{c+n}$.

5.8. Mind-changes and teams. In this section we will consider teams of machines which have a bounded number of mind-changes. All of the machines have the same bound. Recall the definition of consensus value from Lemma 5.31 as a value that shows up at least $a$ times in the list of outputs at time $t$.

We will start with analogues of Lemma 5.31.

OBsERvation 5.42. [1,q]SUBSEQ-EX $\subseteq \subseteq[a, a q] \mathrm{SUBSEQ}_{c}-\mathrm{SX}_{c}$ for all $q, a \geq 1$ and $c \geq 0$.

LEMma 5.43. $[a, b]$ SUBSEQ-EX $c \subseteq[1,\lfloor b / a\rfloor]$ SUBSEQ-EX ${ }_{b(c+1)-1}$ for every $1 \leq a \leq b$ and $c \geq 0$.

Proof. This follows from the second part of the proof of Lemma 5.31, noting that each of the machines $N_{1}, \ldots, N_{q}$ might make a new conjecture any time any one of the $Q_{i}$ does, but not at any other time.

Notice that the previous two results do not give us that

$$
[a, b] \mathrm{SUBSEQ}_{\mathrm{EX}}=[1,\lfloor b / a\rfloor] \mathrm{SUBSEQ}_{c}-\mathrm{EX}_{c}
$$

as in Lemma 5.31. (See Appendix A for the analogue of Lemma 5.43 for EX.)

Corollary 5.44. If $\frac{a}{b}>\frac{1}{2}$ then $[a, b]$ SUBSEQ-EX $c \subseteq$ SUBSEQ-EX $_{b(c+1)-1}$.

TheOREM 5.45. SUBSEQ-EX S(c+1)-1 $\subseteq[a, a q]$ SUBSEQ-EX for $_{c}$ all $a, q \geq 1$ and $c \geq 0$.

Proof. Divide the $a q$ team learners into $q$ groups $G_{1}, \ldots, G_{q}$ of $a$ learners each. Suppose we are given some learner $M$ with some $A$ on the tape. The first time $M$ outputs a conjecture $k_{1}$, the machines in $G_{1}$ (and no others) start outputting $k_{1}$. The next time $M$ changes its mind and outputs a new conjecture $k_{2} \neq k_{1}$, only the machines in $G_{2}$ start outputting $k_{2}$, et cetera. This continues through the groups cyclically. All the machines in some group will eventually output the final DFA output by $M$. There are $q$ groups, and so each team machine makes a $1 / q$ fraction of the conjectures made by $M$. If $M$ makes at most $q(c+1)-1$ mind-changes, then it makes at most $(c+1) q$ conjectures, and so each team machine makes at most $c+1$ conjectures with at most $c$ mindchanges.

From here on out, we will work with teams of the form $[1, b]$. The next two results complement each other. 
Corollary 5.46. SUBSEQ-EX ${ }_{b(c+1)-1} \subseteq[1, b]$ SUBSEQ-EX $_{c}$ for all $b \geq 1$ and $c \geq 0$.

TheOREM 5.47. SUBSEQ-EX ${ }_{b(c+1)} \nsubseteq[1, b]$ SUBSEQ-EX for any $b \geq 1$ and $c \geq 0$.

Proof. We prove that $\mathcal{C}_{b(c+1)} \notin[1, b]$ SUBSEQ-EX by $_{c}$ building a language $A \in \mathcal{C}_{b(c+1)}$ to diagonalize against all $b$ machines. We start by leaving $A$ empty until one of the machines conjectures a DFA for $\emptyset$. Then we add a string to $A$ to render this conjecture incorrect. (The string must of course be long enough so that the machine conjectures the DFA before seeing the string.) Whenever a machine conjectures a DFA for a finite language, we add an appropriately long string to $A$ that is not in the conjectured language. After breaking the $b(c+1)$ conjectures, we will have added at most $b(c+1)$ elements to $A$, so it is in $\mathcal{C}_{b(c+1)}$.

TheOREM 5.48. For all $b \geq 1,[1, b]$ SUBSEQ-EX ${ }_{0} \subseteq$ SUBSEQ-EX $_{2 b-2}$ and $[1, b]$ SUBSEQ-EX ${ }_{c} \subseteq$ SUBSEQ-EX $2 b(c+1)-3$ for all $c \geq 1$.

Proof. We are given $b$ machines team-learning SUBSEQ $(A)$ and outputting at most $c+1$ conjectures each. For $n=1,2,3, \ldots$ we output the DFA (if there is one) that recognizes the $\subseteq$-minimum language among the machines' past outputs that are consistent with the data so far. That is, for each $n$ we output $F$ iff

1. $F$ is an output of one of the $b$ machines running within $n$ steps (not necessarily the most recent output of that machine),

2. SUBSEQ $\left(A \cap \Sigma^{\leq n}\right) \subseteq L(F)$ (that is, $F$ is consistent with the data), and

3. $L(F) \subseteq L(G)$ for any $G$ satisfying items 1 and 2 above.

We'll call such an $F$ good (at time $n$ ). If a good $F$ exists, it is clearly unique. If no good $F$ exists, then we default (in the same sense as in the proof of Theorem 5.36). We can assume for simplicity that at most one of the $b$ machines makes a new conjecture at a time.

Clearly, for all large enough $n$, the correct DFA will be good, and so we will eventually output it forever. To count the number of mind-changes we make, suppose that at some point our current conjecture is some good DFA $F$. We may change our mind away from $F$ for one of two reasons:

finding an inconsistency: we've discovered that $F$ is inconsistent with the data (violating item 2 above) and another good $G$ exists, or

finding something better: $F$ is still consistent, but a good $G$ appears such that $L(G) \subset L(F)$.

Let $G_{0}, G_{1}, G_{2}, \ldots, G_{k}$ be the chronological sequence of DFAs we output, excluding DFAs that equal their immediate predecessors (so $G_{i+1} \neq G_{i}$ for all $0 \leq$ $i<k$ ). So we make exactly $k$ mind-changes for some $k$. Let $V=\left\{G_{0}, \ldots, G_{k}\right\}$ be the set of all DFAs that we output, and let $m=|V|$. We only make conjectures that the team machines make, so $m \leq b(c+1)$. We build a directed graph with vertex set $V$ as follows: For each $0 \leq i<k$, we draw a new directed edge $G_{i} \rightarrow G_{i+1}$ from $G_{i}$ to $G_{i+1}$ representing the mind-change. We color this edge red if the mind-change results from finding $G_{i}$ to be inconsistent, and we color it blue if the mind-change occurs because $G_{i+1}$ is better than $G_{i}$. Note 
that $L\left(G_{i+1}\right) \subset L\left(G_{i}\right)$ if the edge is blue and $L\left(G_{i+1}\right) \nsubseteq L\left(G_{i}\right)$ if the edge is red. Let $R$ be the set of red edges and $B$ the set of blue edges. The sequence of conjectures we make then forms a directed path $p=G_{0} \rightarrow G_{1} \rightarrow \cdots \rightarrow G_{k}$ through the directed graph $(V, R \cup B)$. The path $p$ may visit the same vertex several times. We'll say that the red degree of a vertex $G_{i}$ is the outdegree of $G_{i}$ in the directed graph $(V, R)$, and the blue degree of $G_{i}$ is the indegree of $G_{i}$ in the directed graph $(V, B)$. Our total number $k$ of mind-changes is clearly $|R|+|B|$.

If we find an inconsistency with some $G_{i}$, then we never output $G_{i}$ again. Thus each vertex in $V$ has red degree at most 1 . We never find an inconsistency with the correct team learner's final (correct) output, and so our last conjecture $G_{k}$ has red degree 0 . We therefore have $|R| \leq m-1$.

Suppose that we conjecture some $G_{i}$, change our mind at least once, then conjecture $G_{i}$ again later. We claim that any conjecture $G_{i^{\prime}}$ we make in the interim must satisfy $L\left(G_{i^{\prime}}\right) \subseteq L\left(G_{i}\right)$, and the return to $G_{i}$ follows a red edge:

Claim. Suppose $G_{i} \rightarrow G_{i+1} \rightarrow \cdots \rightarrow G_{j-1} \rightarrow G_{j}=G_{i}$ is a cycle in $p$ of length $j-i \geq 2$. Then $L\left(G_{i^{\prime}}\right) \subseteq L\left(G_{i}\right)$ for all $i \leq i^{\prime} \leq j$. Furthermore, $G_{j-1} \rightarrow G_{j}$ is a red edge.

Proof of the Claim. Since the cycle starts and ends with $G_{i}, G_{i}$ is known and consistent with the data throughout the cycle. This means that any $G_{i^{\prime}}$ conjectured in the interim (being good at the time of conjecture) must satisfy $L\left(G_{i^{\prime}}\right) \subseteq L\left(G_{i}\right)$ by the $\subseteq$-minimality of $L\left(G_{i^{\prime}}\right)$. It follows immediately that the return to $G_{j}=G_{i}$ can only come from following a red edge, i.e., finding an inconsistency, for otherwise we would have $L\left(G_{j}\right) \subset L\left(G_{j-1}\right)$ (and thus $\left.L\left(G_{j-1}\right) \nsubseteq L\left(G_{j}\right)=L\left(G_{i}\right)\right)$. End of Proof of Claim

It follows from the Claim that each vertex in $V$ has blue degree at most 1 , and that our very first conjecture $G_{0}$ has blue degree 0 . Thus $|B| \leq m-1$. Combining this with the bound on $|R|$ gives us $|R|+|B| \leq 2 m-2 \leq 2 b(c+1)-2$ mind-changes. This is enough for the $c=0$ case of the theorem.

Now assuming $c \geq 1$, we will shave off another mind-change. We are done if $|R|<m-1$, so suppose $|R|=m-1$. This can happen only if all the vertices of $V$ have red degree 1 except $G_{k}$ - our final conjecture-which has red degree 0 . First, suppose $G_{0} \neq G_{k}$. Then $G_{0}$ has red degree 1 , and so at some point we follow a red edge from $G_{0}$ to some other $H$. Since $L(H) \nsubseteq L L\left(G_{0}\right)$ because of the red edge, the Claim implies that we have not conjectured $H$ before (otherwise, consider the initial cycle $G_{0} \rightarrow \cdots \rightarrow H \rightarrow \cdots \rightarrow G_{0}$ and apply the Claim). And so, also by the Claim, $H$ must have blue degree 0 , because we first encounter $H$ through a red edge. So we have two vertices $\left(G_{0}\right.$ and $\left.H\right)$ with blue degree 0 , and thus $|B| \leq m-2$, and we have at most $2 m-3 \leq 2 b(c+1)-3$ mind-changes.

Now suppose $G_{0}=G_{k}$. Then it is possible that $|R|+|B|=2 m-2$, but we will see that in this case, $m<b(c+1)$, and thus our algorithm still uses at most $2 b(c+1)-3$ mind-changes. Let $M$ be one of the $b$ team machines that eventually outputs the correct DFA (i.e., $G_{k}$ ) forever. If one of the $b$ machines other than $M$ outputs $G_{k}$, or if $M$ outputs $G_{k}$ at some point before changing its mind, then the $b$ machines collectively make strictly fewer than $b(c+1)$ distinct conjectures, and so $m<b(c+1)$. So we can assume that $G_{k}$ appears only as 
the final conjecture made by $M$. We claim that $V$ does not contain any other conjecture made by $M$ except $G_{k}$, which shows that $m<b(c+1)$. If $M$ makes a conjecture $H \neq G_{k}$, it does so before it ever outputs $G_{k}$, and so we know about $H$ before we output $G_{0}$ as our very first conjecture. If $H$ is inconsistent with the data when we first output $G_{0}$, then we never output $H$, and hence $H \notin V$ and we are done. But otherwise, $H$ has been consistent with the data from the time we first discovered it, i.e., before we first discover $G_{0}$. In this case, we would have output some good DFA (perhaps $H$ ) before discovering $G_{0}$, contradicting the fact that $G_{0}$ is our initial conjecture. Thus we never output $H$, which proves the claim and the theorem.

Theorem 5.48 is tight.

Theorem 5.49. For all $b>1,[1, b]$ SUBSEQ-EX $_{0} \nsubseteq$ SUBSEQ-EX $_{2 b-3}$ and $[1, b] \mathrm{SUBSEQ}_{-\mathrm{EX}_{c}} \not \mathrm{SUBSEQ}_{\mathrm{SOX}} 2 b(c+1)-4$ for all $c \geq 1$.

Proof. We'll only prove the case where $c \geq 1$. The $c=0$ case is easier and only slightly different.

Let $f: \mathbb{N}^{+} \rightarrow \mathbb{N}$ be any map. For any $j \in \mathbb{N}$, define a $j$-bump of $f$ to be any nonempty, finite, maximal interval $[x, y] \subseteq \mathbb{N}^{+}$such that $f(t)>j$ for all $x \leq t \leq y$. Define the language

$$
A_{f}:=\left\{\left(0^{t} 1^{t}\right)^{f(t)}: t \in \mathbb{N}^{+}\right\}
$$

Observe that, if $\limsup _{t \rightarrow \infty} f(t)=\ell<\infty$, then $f$ has finitely many $\ell$-bumps and $R_{\ell} \subseteq \operatorname{SUBSEQ}\left(A_{f}\right) \subseteq^{*} R_{\ell}$, where $R_{\ell}=\left(0^{*} 1^{*}\right)^{\ell}$ as in Definition 4.8 .

Now fix $b>1$ and $c \geq 1$. We say that $f$ is $\operatorname{good}$ if

- $f(1)=b$ and $0 \leq f(t) \leq b$ for all $t \geq 1$,

- $f$ has at most $c$ many 0-bumps,

- $f$ has at most $c+1$ many $\ell$-bumps, where $\ell=\lim \sup _{t} f(t)$, and

- if $(\exists t)[f(t)=0]$ then $\limsup _{t} f(t) \leq b-1$.

We define the class

$$
\mathcal{T}_{b, c}:=\left\{A_{f}: f \text { is good }\right\},
$$

and show that $\mathcal{T}_{b, c} \in[1, b]$ SUBSEQ-EX ${ }_{c}-$ SUBSEQ-EX $_{2 b(c+1)-4}$.

To see that $\mathcal{T}_{b, c} \in[1, b]$ SUBSEQ-EX ${ }_{c}$, we define learners $Q_{1}, \ldots, Q_{b}$ acting as follows with $A_{f}$ on their tapes for some good $f$ : Each learner examines its tape enough to determine $f(1), f(2), \ldots$ For $1 \leq j \leq b-1$, learner $Q_{j}$ goes on the assumption that $\limsup _{t} f(t)=j$. Each time it notices a new $j$-bump $[x, y]$ of $f$, it assumes that $[x, y]$ is the last $j$-bump it will see and so starts outputting a DFA for

$$
R_{j} \cup \operatorname{SUBSEQ}\left(A_{f} \cap\left\{\left(0^{t} 1^{t}\right)^{k}: t \leq y \wedge k \leq b\right\}\right) .
$$

which captures all the elements of $A_{f}-R_{j}$ seen so far. Let $\ell=\lim \sup _{t} f(t)$. If $1 \leq \ell \leq b-1$, then $Q_{\ell}$ will see at most $c+1$ many $\ell$-bumps of $f$ and so make at most $c+1$ conjectures, the last one being correct.

The learner $Q_{b}$ behaves a bit differently: It immediately starts outputting the DFA for $R_{b}$, and does this until it (ever) finds a $t$ with $f(t)=0$. It then proceeds on the assumption that $\lim \sup _{t} f(t)=0$ and acts similarly to the other learners. Again, let $\ell=\limsup _{t} f(t)$. Since $f$ is good, if there is a $t$ such that $f(t)=0$, 
then $\ell \leq b-1$ and so all possible values of $\ell$ are covered by the learners. If $\ell=0$, then since there are only $c$ many 0 -bumps, $Q_{b}$ will be correct after at most $c+1$ conjectures. If $\ell=b$, then $\operatorname{SUBSEQ}\left(A_{f}\right)=R_{b}$, and since $f$ is good, $Q_{b}$ will never revise its initial conjecture of $R_{b}$. This establishes that $\mathcal{T}_{b, c} \in[1, b]$ SUBSEQ-EX ${ }_{c}$.

To show that $\mathcal{T}_{b, c} \notin \mathrm{SUBSEQ}-\mathrm{EX}_{2 b(c+1)-4}$, let $M$ be a learner that correctly learns SUBSEQ $\left(A_{f}\right)$ for every good $f$. We now describe a particular good $f$ that forces $M$ to make at least $2 b(c+1)-3$ mind-changes.

For $t=1,2,3, \ldots$, we first let $f(t)=b$ until $M$ outputs a DFA for $R_{b}$. Then we make $f(t)=b-1$ until $M$ outputs a DFA $F$ such that $R_{b-1} \subseteq L(F) \subseteq{ }^{*} R_{b-1}$, at which point we start making $f(t)=b$ again, et cetera. The value of $f(t)$ alternates between $b$ and $b-1$, forcing a mind-change each time, until $f(t)=b-1$ and there are $c+1$ many $(b-1)$-bumps of $f$. Then $f$ starts alternating between $b-1$ and $b-2$ in a similar fashion until there are $c+1$ many $(b-2)$-bumps, et cetera. These alternations continue until $f(t)=0$ and there are $c$ many 0 -bumps of $f$ included in the interval $[1, t]$. Thus far, $M$ has needed to make $2 c+1$ many conjectures for each of the first $b-1$ many alternations, plus $2 c$ conjectures for the 1,0 alternation, for a total of $(b-1)(2 c+1)+2 c=2 b c+b-1$ many conjectures.

Now we let $f(t)$ slowly increase from 0 through to $b-1$, forcing a new conjecture with each step, until we settle on $b-1$. This adds $b-1$ more conjectures for a grand total of $2 b c+2(b-1)=2 b(c+1)-2$ conjectures, or $2 b(c+1)-3$ mind-changes.

5.9. All three modifications. Finally, we consider teams of machines which are allowed to have anomalies, but have a bounded number of mind-changes.

TheOrEm 5.50. $[a, b] \mathrm{SUBSEQ}_{\mathrm{EX}}^{k} \subseteq[a, b] \mathrm{SUBSEQ}_{c}-\mathrm{EX}_{c}$ for all $c, k \geq 0$ and $1 \leq a \leq b$.

Proof. This follows from the proof of Theorem 5.36. We apply the algorithm there to each of the $b$ machines.

S6. Rich classes. Are there classes in SUBSEQ-EX containing languages of arbitrary complexity? Yes, trivially.

Proposition 6.1. There is a $\mathcal{C} \in \mathrm{SUBSEQ}_{0} \mathrm{EX}_{0}$ such that for all $A \subseteq \mathbb{N}$, there is a $B \in \mathcal{C}$ with $B \equiv_{\mathrm{T}} A$.

ProOF. Let

$$
\mathcal{C}=\left\{A \subseteq \Sigma^{*}:|A|=\infty \wedge\left(\forall x, y \in \Sigma^{*}\right)[x \in A \wedge|x|=|y| \rightarrow y \in A]\right\} .
$$

That is, $\mathcal{C}$ is the class of all infinite languages, membership in whom depends only on a string's length.

For any $A \subseteq \mathbb{N}$, define

$$
L_{A}= \begin{cases}\Sigma^{*} & \text { if } A \text { is finite } \\ \bigcup_{n \in A} \Sigma^{=n} & \text { otherwise. }\end{cases}
$$

Clearly, $L_{A} \in \mathcal{C}$ and $A \equiv_{\mathrm{T}} L_{A}$. Furthermore, $\operatorname{SUBSEQ}(L)=\Sigma^{*}$ for all $L \in \mathcal{C}$, and so $\mathcal{C} \in \mathrm{SUBSEQ}-\mathrm{EX}_{0}$ witnessed by a learner that always outputs a DFA for $\Sigma^{*}$. 
In Proposition 4.18 we showed that REG $\in$ SUBSEQ-EX. Note that the $A \in$ REG are trivial in terms of computability, but the languages in SUBSEQ(REG) can be rather complex (large obstruction sets, arbitrary $\preceq$-closed sets). By contrast, in Proposition 6.1, we show that there can be $\mathcal{A} \in$ SUBSEQ-EX of arbitrarily high Turing degree but $\operatorname{SUBSEQ}(\mathcal{A})$ is trivial. Can we obtain classes $\mathcal{A} \in \operatorname{SUBSEQ}-E X$ where $A \in \mathcal{A}$ has arbitrary Turing degree and $\operatorname{SUBSEQ}(\mathcal{A})$ has arbitrary $\preceq$-closed sets independently? Of course, if $\operatorname{SUBSEQ}(A)$ is finite, then $A$ must be finite and hence computable. Aside from this obvious restriction, the answer to the above question is yes.

Definition 6.2. A class $\mathcal{C}$ of languages is rich if for every $A \subseteq \mathbb{N}$ and $\preceq$ closed $S \subseteq \Sigma^{*}$, there is a $B \in \mathcal{C}$ such that $\operatorname{SUBSEQ}(B)=S$ and, provided $A$ is computable or $S$ is infinite, $B \equiv_{\mathrm{T}} A$.

Definition 6.3. Let $\mathcal{G}$ be the class of all languages $A \subseteq \Sigma^{*}$ for which there exists a length $c=c(A) \in \mathbb{N}$ (necessarily unique) such that

1. $A \cap \Sigma^{=c}=\emptyset$,

2. $A \cap \Sigma^{=n} \neq \emptyset$ for all $n<c$, and

3. $\operatorname{os}(A)=\operatorname{os}\left(A \cap \Sigma^{\leq c+1}\right) \cap \Sigma^{\leq c}$.

We'll show that $\mathcal{G} \in \mathrm{SUBSEQ} \mathrm{EX}_{0}$ and that $\mathcal{G}$ is rich.

Proposition 6.4. $\mathcal{G} \in \mathrm{SUBSEQ}_{0}$.

Proof. Consider a learner $M$ acting as follows with a language $A$ on its tape:

1. Let $c$ be least such that $A \cap \Sigma^{=c}=\emptyset$ (assuming $c$ exists).

2. Compute $O=\operatorname{os}\left(A \cap \Sigma^{\leq c+1}\right) \cap \Sigma^{\leq c}$. (If $A \in \mathcal{G}$, then $O=\operatorname{os}(A)$ by definition.)

3. Use $O$ to compute the least index $k$ such that $L\left(F_{k}\right)$ is $\preceq$-closed and $\operatorname{os}\left(L\left(F_{k}\right)\right)=O$. (If $A \in \mathcal{G}$, then we have $L\left(F_{k}\right)=\operatorname{SUBSEQ}(A)$, because $O=\operatorname{os}(A)=\operatorname{os}(\operatorname{SUBSEQ}(A))$.)

4. Output $k$ repeatedly forever.

It is evident that $M$ learns every language in $\mathcal{G}$ with no mind-changes.

The next few propositions show that $\mathcal{G}$ is big enough.

Definition 6.5. Let $S \subseteq \Sigma^{*}$ be any $\preceq$-closed set.

1. Say that a string $x$ is $S$-special if $x \in S$ and $S \cap\left\{y \in \Sigma^{*}: x \preceq y\right\}$ is finite.

2. Say that a number $n \in \mathbb{N}$ is an $S$-coding length if $n>|y|$ for all $S$-special $y$ and $n \geq|z|$ for all $z \in \operatorname{os}(S)$.

The next proposition implies that $S$-coding lengths exist for any $S$.

Proposition 6.6. Any $\preceq$-closed $S$ has only finitely many $S$-special strings.

PRoof. This follows from the fact, first proved by Higman [22], that $\left(\Sigma^{*}, \preceq\right)$ is a well-quasi-order (wqo). That is, for any infinite sequence $x_{1}, x_{2}, \ldots$ of strings, there is some $i<j$ such that $x_{i} \preceq x_{j}$. A standard result of well-quasi-order theory, proved using techniques from Ramsey theory, gives a stronger fact: Every infinite sequence $x_{1}, x_{2}, \ldots$ of strings contains an infinite monotone subsequence

$$
x_{i_{1}} \preceq x_{i_{2}} \preceq \cdots,
$$


where $i_{1}<i_{2}<\cdots$.

Suppose that some $S$ has infinitely many $S$-special strings $s_{1}, s_{2}, \ldots$ with all the $s_{i}$ distinct. Then $S$ includes an infinite monotone subsequence $s_{i_{1}} \prec s_{i_{2}} \prec \cdots$ of $S$-special strings, but then $s_{i_{1}}$ clearly cannot be $S$-special. Contradiction. $\dashv$

Corollary 6.7. S-coding lengths exist for any $\preceq$-closed $S$.

Definition 6.8. Let $\mathcal{G}^{\prime}$ be the class of all $A \subseteq \Sigma^{*}$ that have the following properties (setting $S=\operatorname{SUBSEQ}(A)$ ):

1. A contains all $S$-special strings, and

2. there exists a (necessarily unique) $S$-coding length $c$ for which the following hold:
(a) $A \cap \Sigma^{=c}=\emptyset$,
(b) $A \cap \Sigma^{=n} \neq \emptyset$ for all $n<c$, and
(c) $A \cap \Sigma^{=c+1}=S \cap \Sigma^{=c+1}$.

Proposition 6.9. $\left\{A \subseteq \Sigma^{*}: A\right.$ is $\preceq$-closed and finite $\} \subseteq \mathcal{G}^{\prime} \subseteq \mathcal{G}$.

Proof. For the first inclusion, it is easy to check that the criteria of Definition 6.8 hold for any finite $\preceq$-closed $A$ if we let $c$ be least such that $A \subseteq \Sigma^{<c}$.

For the second inclusion, suppose $A \in \mathcal{G}^{\prime}$, and let $c$ satisfy the conditions of Definition 6.8 for $A$. It remains to show that

$$
\operatorname{os}(A)=\operatorname{os}\left(A \cap \Sigma^{\leq c+1}\right) \cap \Sigma^{\leq c} .
$$

Set $S=\operatorname{SUBSEQ}(A)$. Since $c$ is an $S$-coding length, we have os $(A)=\operatorname{os}(S) \subseteq$ $\Sigma \leq c$.

Let $x$ be some string in $\operatorname{os}(A)$. Then $x \notin S$, but $y \in S$ for every $y \prec x$. Consider any $y \prec x$.

- If $y$ is $S$-special, then $y \in A$ (since $A$ contains all $S$-special strings), and since $|y|<|x| \leq c$, we have $y \in A \cap \Sigma^{\leq c+1}$, and so $y \in \operatorname{SUBSEQ}\left(A \cap \Sigma^{\leq c+1}\right)$.

- If $y$ is not $S$-special, then there are arbitrarily long $z \in S$ with $y \preceq z$. In particular there is a $z \in S \cap \Sigma^{=c+1}$ such that $y \preceq z$. But then $z \in A \cap \Sigma^{=c+1}$ (because $\left.A \in \mathcal{G}^{\prime}\right)$, which implies $y \in \operatorname{SUBSEQ}\left(A \cap \Sigma^{\leq c+1}\right)$.

In either case, we have shown that $x$ is not in $\operatorname{SUBSEQ}\left(A \cap \Sigma^{\leq c+1}\right)$, but every $y \prec x$ is in $\operatorname{SUBSEQ}\left(A \cap \Sigma^{\leq c+1}\right)$. This means exactly that $x \in \operatorname{os}\left(A \cap \Sigma^{\leq c+1}\right)$, and since $|x| \leq c$, we have the forward containment in (4).

Conversely, suppose that $|x| \leq c$ and $x \in \operatorname{os}\left(A \cap \Sigma^{\leq c+1}\right)$. Then $x \notin A \cap \Sigma^{\leq c+1}$ but $(\forall y \prec x)(\exists z \in A \cap \Sigma \leq c+1)[y \preceq z]$. Thus, $x \notin A$ but $(\forall y \prec x)(\exists z \in A)[y \preceq z]$. That is, $x \in \operatorname{os}(A)$.

THEOREM 6.10. $\mathcal{G}^{\prime}$ is rich. In fact, there is a learner $M$ such that $M$ learns every language in $\mathcal{G}^{\prime}$ without mind-changes, and for every $A$ and infinite $S, M$ learns some $B \in \mathcal{G}^{\prime}$ satisfying Definition 6.2 while also writing the characteristic function of $A$ on a separate one-way write-only output tape.

Proof. Given $A$ and $S$ as in Definition 6.2 , we define

$$
L(A, S):=S \cap\left(\Sigma^{<c} \cup \bigcup_{n \in \mathbb{N}} \Sigma^{=c+2 n+1} \cup \bigcup_{n \in A} \Sigma^{=c+2 n+2}\right),
$$

where $c$ is the least $S$-coding length. 
Set $B=L(A, S)$, and let $c$ be the least $S$-coding length.

We must first show that $S=\operatorname{SUBSEQ}(B)$ and that $B \in \mathcal{G}^{\prime}$. We have two cases: $S$ is finite or $S$ is infinite. First suppose that $S$ is finite. Then every string in $S$ is $S$-special, and so by the definition of $S$-coding length, we have $S \subseteq \Sigma^{<c}$. Thus we clearly have $B=S=\operatorname{SUBSEQ}(B) \in \mathcal{G}^{\prime}$ by Proposition 6.9.

Now suppose $S$ is infinite. Since $B \subseteq S$ and $S$ is $\preceq$-closed, to get $S=$ $\operatorname{SUBSEQ}(B)$ it suffices to show that $S \subseteq \operatorname{SUBSEQ}(B)$. Let $x$ be any string in $S$.

- If $x$ is $S$-special, then $x \in \Sigma^{<c}$, by the definition of $S$-coding length. It follows that $x \in B$, and so $x \in \operatorname{SUBSEQ}(B)$.

- If $x$ is not $S$-special, then there is a string $z \in S$ such that $x \preceq z$ and $|z| \geq c+2|x|+1$. By removing letters one at a time from $z$ to obtain $x$, we see that at some point there must be a string $y$ such that $x \preceq y \preceq z$ and $|y|=c+2|x|+1$. Thus $y \in S$, and, owing to its length, $y \in B$ as well. Therefore we have $x \in \operatorname{SUBSEQ}(B)$.

Now that we know that $S=\operatorname{SUBSEQ}(B)$, it is straightforward to verify that $B \in \mathcal{G}^{\prime}$. We showed above that $B$ contains all $S$-special strings. The value $c$ clearly satisfies the rest of Definition 6.8. For example, because $S$ has strings of every length, we have $B \cap \Sigma^{=n}=S \cap \Sigma^{=n} \neq \emptyset$ for all $n<c$.

It is immediate by the definition that $B \leq_{\mathrm{T}} A$, because $S$ is regular. We now describe the learner $M$, which will witness that $A \leq_{\mathrm{T}} B$ as well, provided $S$ is infinite. $M$ behaves exactly as in the proof of Proposition 6.4, except that for $n=0,1,2, \ldots$ in order, $M$ appends a 1 to the string on its special output tape if $B \cap \Sigma^{=c+2 n+2} \neq \emptyset$, and it appends a 0 otherwise. If $S$ is infinite, then $S$ contains strings of every length, and so $M$ will append a 1 for $n$ if and only if $n \in A$. (If $S$ is finite, then $M$ will write all zeros.)

Corollary 6.11. $\mathcal{G}$ is rich.

S7. Open questions. We have far from fully explored the different ways we can combine teams, mind-changes, and anomalies. For example, for which $a, b, c, d, e, f, g$ is $[a, b] \mathrm{SUBSEQ}-\mathrm{EX}_{c}^{d} \subseteq[e, f] \mathrm{SUBSEQ}-\mathrm{EX}_{g}^{h}$ ? This problem has been difficult in the standard case of EX, though there have been some very interesting results $[14,8]$. The setting of SUBSEQ-EX may be easier since all the machines that are output are total and their languages have easily discernible properties. Of particular interest is the four-parameter problem of mind-changes versus teams: For which $b, c, m, n$ is $[1, m]$ SUBSEQ-EX $_{b} \subseteq[1, n]$ SUBSEQ-EX $_{c}$ ? This is interesting in that our current partial results do not completely match those in [32] for EX.

We also have not studied in depth different ways of combining variants of the alternate ways of learning SUBSEQ-EX given in Section 5.2. For example, BC learning of co-c.e. indices for $\operatorname{SUBSEQ}(A)$ is equivalent to SUBSEQ-EX (Proposition 5.9), as is $\mathrm{BC}$ learning of $\mathrm{DFAs}$ for $\operatorname{SUBSEQ}(A)$ with finite anomalies (Proposition 5.19), but is the combination of the two-BC learning of co-c.e. indices for SUBSEQ $(A)$ with finite anomalies - equivalent to SUBSEQ-EX? We suspect not. What about combining mind-changes or teams with learning other devices or learning from one-sided data? 
One could also combine the two notions of queries with SUBSEQ-EX and its variants. The two notions are allowing queries about the set $[19,17,15]$ and allowing queries to an undecidable set $[12,26]$.

Acknowledgments. The authors would like to thank Walid Gomma and Semmy Purewal for proofreading and helpful discussions. We also thank two anonymous referees for many helpful suggestions, including Observation 5.14. Finally, we thank Sanjay Jain for informing us of Theorem A.1 and providing a proof, and we thank Mahe Velauthapillai for informing us of the improvements in [25] to Theorem A.1.

Appendix A. Mind-changes and teams for EX. The following result is not in the literature. It is the analogue to Lemma 5.43 for EX, which we include for completeness. It was proven by Sanjay Jain [23], whose proof sketch is below.

Theorem A.1 (Jain [23]). For all $a, b$, and $c$,

$$
[a, b] \mathrm{EX}_{c} \subseteq[1, d] \mathrm{EX}_{b(c+1)-1},
$$

where $d=\lfloor b / a\rfloor$.

Proof sketch. Let $C \in[a, b] \mathrm{EX}_{c}$ via machines $M_{1}, \ldots, M_{b}$. We construct $N_{1}, \ldots, N_{d}$ to show that $C \in[1, d] \mathrm{EX}_{b(c+1)-1}$. Assume without loss of generality that the programs output by different $M_{i}$ are always distinct (this can be ensured by padding).

Let $\varphi_{0}, \varphi_{1}, \varphi_{2}, \ldots$ be a standard listing of all partial computable functions. If one of the $M_{i}$ or $N_{i}$ outputs $j$, then this means that it thinks the function is $\varphi_{j}$.

Assume the input to the $d$ machines is the function $f$, so they are getting $f(0), f(1), \ldots$ We feed them to the $M_{1}, \ldots, M_{b}$, observe the output, and carefully output some of the results.

We say that a program $p$ output by some machine $M_{i}$ is spoiled if either it is seen to be convergently wrong, or there is a later mind change by $M_{i}$.

Initially, all of the $N_{1}, \ldots, N_{d}$ are available, and none has output a program. We dovetail the following two steps forever:

1. If at any stage there is a set $S$ of $a$ unspoiled programs which have not been assigned to a machine from $N_{1}, \ldots, N_{d}$, then assign $S$ to an available machine from $N_{1}, \ldots, N_{d}$, which then becomes unavailable (we can argue that there will be such an available machine, as there are at most $b$ unspoiled programs at any stage). The program output by this machine will be for the function which, on input $x$, dovetails all $\varphi_{j}(x)$ for $j \in S$ and outputs the first answer that converges.

2. If a program from the set of programs $S$ assigned to $N_{i}$ gets spoiled, then $N_{i}$ becomes available, and all the unspoiled programs in the set $S$ assigned to $N_{i}$ become unassigned.

The result easily follows since the following two facts hold:

1. Final programs output by $N_{i}$ 's do not make a convergent error, and at least one of the $N_{i}$ 's has a correct program in its set of assigned programs.

2. Each mind change of any $N_{i}$ corresponds to a corresponding spoiling of some program output by some machine in $M_{1}, \ldots, M_{b}$. Thus, in total 
there are at most $b(c+1)-1$ mind changes. (Actually there are much fewer, as we start with at least $a$ programs being assigned, and we could also do assignments so that not everything comes to the same $N_{i}$.)

As was suggested in its proof, this inclusion is not tight. Jain, Sharma, \& Velauthapillai [25] have obtained (among other things) $[3,6] \mathrm{EX}_{0}=[1,2] \mathrm{EX}_{0}$, which is better than Theorem A.1 with $a=3, b=6$, and $c=0$.

\section{REFERENCES}

[1] A. Ambainis, S. Jain, and A. Sharma, Ordinal mind change complexity of language identification, Theoretical Computer Science, vol. 220 (1999), no. 2, pp. 323-343.

[2] D. Angluin, Inductive inference of formal languages from positive data, Information and Control, vol. 45 (1980), no. 2, pp. 117-135.

[3] G. BAliga and J. CASE, Learning with higher order additional information, Proceedings of the 5th international workshop on algorithmic learning theory, Springer-Verlag, 1994, pp. 64-75.

[4] J. M. BARzDin, Two theorems on the limiting synthesis of functions, Theory of algorithms and programs (J. Barzdin, editor), Latvian State University, Riga, U.S.S.R., 1974, pp. $82-88$.

[5] L. BLum and M. Blum, Towards a mathematical theory of inductive inference, Information and Control, vol. 28 (1975), pp. 125-155.

[6] J. Case, S. Jain, and S. N. Manguelle, Refinements of inductive inference by Popperian and reliable machines, Kybernetika, vol. 30-1 (1994), pp. 23-52.

[7] J. CASE and C. H. SмIтH, Comparison of identification criteria for machine inductive inference, Theoretical Computer Science, vol. 25 (1983), pp. 193-220.

[8] R. Daley, B. Kalyanasundaram, and M. Velauthapillai, Breaking the probability 1/2 barrier in FIN-type learning, Journal of Computer and System Sciences, vol. 50 (1995), pp. 574-599.

[9] Y. L. Ershov, S. S. Goncharov, A. Nerode, and J. B. Remmel (editors), Handbook of recursive mathematics, Elsevier North-Holland, Inc., New York, 1998, Comes in two volumes. Volume 1 is Recursive Model Theory, Volume 2 is Recursive Algebra, Analysis, and Combinatorics.

[10] S. Fenner and W. Gasarch, The complexity of learning SUBSEQ(A), Proceedings of the 17th international conference on algorithmic learning theory, Lecture Notes in Artificial Intelligence, vol. 4264, Springer-Verlag, 2006, pp. 109-123.

[11] S. Fenner, W. Gasarch, and B. Postow, The complexity of finding SUBSEQ(A), Theory of Computing Systems, (2008), to appear.

[12] L. Fortnow, S. Jain, W. Gasarch, E. Kinber, M. Kummer, S. Kurtz, M. Pleszkoch, T. Slaman, F. Stephan, and R. Solovay, Extremes in the degrees of inferability, Annals of Pure and Applied Logic, vol. 66 (1994), no. 3, pp. 231-276.

[13] R. Freivalds and C. H. Smith, On the role of procrastination for machine learning, Information and Computation, vol. 107 (1993), no. 2, pp. 237-271.

[14] R. Freivalds, C. H. Smith, and M. Velauthapillai, Trade-off among parameters affecting inductive inference, Information and Computation, vol. 82 (1989), no. 3, pp. 323349.

[15] W. Gasarch, E. Kinber, M. Pleszkoch, C. H. Smith, and T. Zeugmann, Learning via queries, teams, and anomalies, Fundamenta Informaticae, vol. 23 (1995), pp. 67-89, Prior version in Computational Learning Theory (COLT), 1990.

[16] W. Gasarch and A. Lee, Inferring answers to queries, Proceedings of the 10th annual acm conference on computational learning theory, 1997, Journal version to appear in JCSS in 2008, pp. 275-284.

[17] W. Gasarch, M. Pleszkoch, and R. Solovay, Learning via queries to $[+,<]$, this JournaL, vol. 57 (1992), no. 1, pp. 53-81. 
[18] W. Gasarch, M. Pleszkoch, F. Stephan, and M. Velauthapillai, Classification using information, Annals of Mathematics and Artificial Intelligence, vol. 23 (1998), pp. 147-168, Earlier version in Proceedings of the 5th International Workshop on Algorithmic Learning Theory, 1994, 290-300.

[19] W. Gasarch and C. H. Smith, Learning via queries, Journal of the ACM, vol. 39 (1992), no. 3, pp. 649-675, Prior version in Proceedings of the 29th IEEE Symposium on Foundations of Computer Science (FOCS), 1988.

[20] E. M. Gold, Language identification in the limit, Information and Control, vol. 10 (1967), no. 10, pp. 447-474.

[21] J. Hartmanis, Context-free languages and Turing machine computations, Mathematical aspects of computer science (J. T. Schwartz, editor), Proceedings of Symposia in Applied Mathematics, vol. 19, American Mathematical Society, Providence, RI, 1967, pp. 42-51.

[22] A. G. Higman, Ordering by divisibility in abstract algebras, Proceedings of the London Mathematical Society, vol. s3-2 (1952), no. 1, pp. 326-336.

[23] S. JAIN, 2008, personal communication.

[24] S. JAIN and A. Sharma, Computational limits on team identification of languages, Information and Computation, vol. 130 (1996), no. 1, pp. 19-60.

[25] S. Jain, A. Sharma, and M. Velauthapillai, Finite identification of functions by teams with success ratio 1/2 and above, Information and Computation, vol. 121 (1995), no. 2 , pp. 201-213.

[26] M. Kummer and F. Stephan, On the structure of the degrees of inferability, Journal of Computer and System Sciences, vol. 52 (1996), no. 2, pp. 214-238, Prior version in Sixth Annual Conference on Computational Learning Theory (COLT), 1993.

[27] G. Metakides and A. Nerode, Effective content of field theory, Annals of Mathematical Logic, vol. 17 (1979), pp. 289-320.

[28] L. PITt, Probabilistic inductive inference, Journal of the ACM, vol. 36 (1989), no. 2, pp. $383-433$.

[29] L. Piтt and C. H. Smith, Probability and plurality for aggregations of learning machines, Information and Computation, vol. 77 (1988), pp. 77-92.

[30] H. ROGERS, Theory of recursive functions and effective computability, McGrawHill, 1967, Reprinted by MIT Press, 1987.

[31] G. E. SACKS, Higher recursion theory, Perspectives in Mathematical Logic, SpringerVerlag, Berlin, 1990.

[32] C. H. Sмiтн, The power of pluralism for automatic program synthesis, Journal of the ACM, vol. 29 (1982), pp. 1144-1165, Prior version in Proceedings of the 22nd IEEE Symposium on Foundations of Computer Science (FOCS), 1981.

[33] R. I. SOARE, Recursively enumerable sets and degrees, Perspectives in Mathematical Logic, Springer-Verlag, Berlin, 1987.

[34] J. VAn LEEUWEn, Effective constructions in well-partially-ordered free monoids, Discrete Mathematics, vol. 21 (1978), pp. 237-252.

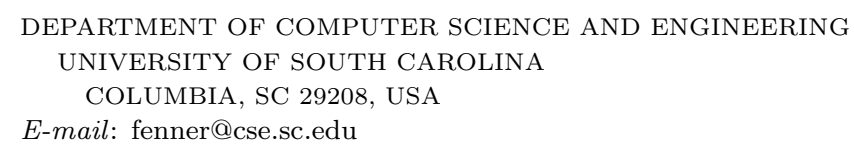

DEPARTMENT OF COMPUTER SCIENCE AND INSTITUTE FOR ADVANCED COMPUTER STUDIES

UNIVERSITY OF MARYLAND AT COLLEGE PARK

COLLEGE PARK, MD 20742, USA

E-mail: gasarch@cs.umd.edu

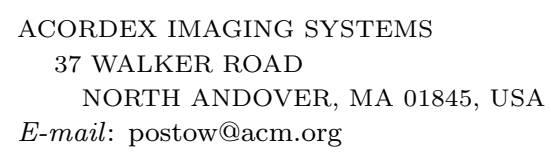

